\title{
Local regularization of the One-Phase Hele-Shaw Flow
}

\author{
Sunhi Choi* David Jerison ${ }^{\dagger}$ and Inwon Kim ${ }^{\ddagger}$
}

March 4, 2008

\begin{abstract}
This article presents a local regularity theorem for the one-phase Hele-Shaw flow. We prove that if the Lipschitz constant of the initial free boundary in a unit ball is small, then for small uniform positive time the solution is smooth. This result improves on our earlier results in [CJK] because it is scale-invariant. As a consequence we obtain existence, uniqueness and regularity properties of global solutions with Lipschitz initial free boundary.
\end{abstract}

\section{Introduction}

For $(x, t) \in \mathbb{R}^{n} \times \mathbb{R}$ (or some subset) let $u(x, t)$ be a viscosity solution (defined in the next section) of the Hele-Shaw problem

$$
\begin{cases}-\Delta u(\cdot, t)=0 & \text { in }\{u>0\} \\ \frac{\partial u}{\partial t}=|D u|^{2} & \text { on } \partial\{u>0\}\end{cases}
$$

where $D u$ denotes the gradient of $u$ in the space variables $x$. We refer to

$$
\Omega_{t}(u)=\{x: u(x, t)>0\} \subset \mathbb{R}^{n}, \quad \Omega(u)=\{(x, t): u>0\} \subset \mathbb{R}^{n+1}
$$

\footnotetext{
*Department of Mathematics, University of Arizona. e-mail: schoi@math.arizona.edu. Partially supported by NSF grant 0713598 .

${ }^{\dagger}$ Department of Mathematics, MIT. e-mail: jerison@math.mit.edu. Partially supported by NSF grant DMS 0244991 .

${ }^{\ddagger}$ Department of Mathematics, UCLA. e-mail: ikim@math.ucla.edu. Partially supported by NSF grant DMS 0627896
} 


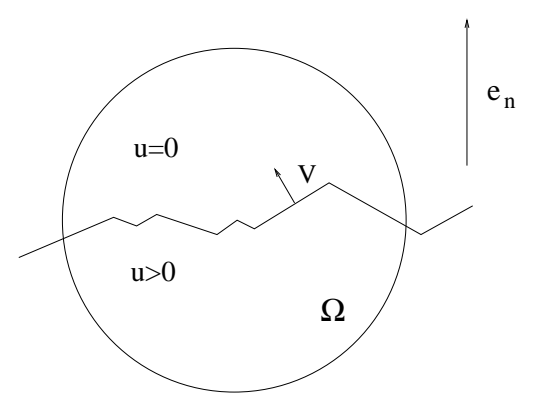

Figure 1: Initial setting of the problem

as the positive phase of $u$ and to

$$
\Gamma_{t}(u)=\partial \Omega_{t}(u), \quad \Gamma(u)=\partial \Omega(u)
$$

as the free boundary of $u$.

If $u$ is smooth up to the free boundary, then the free boundary moves with normal velocity $V=u_{t} /|D u|$, and hence the second equation in (HS) implies that

$$
V=|D u|=-\frac{\partial u}{\partial \nu},
$$

where $\nu$ is the outward spatial normal, with respect to $\Omega_{t}(u)$, on $\Gamma_{t}(u)$. (see Figure 1)

The classical Hele-Shaw problem models an incompressible viscous fluid which occupies part of the space between two parallel, nearby plates, and $u$ is the pressure of the fluid. The short-time existence of classical solutions with initial surface $\Gamma_{0}$ of class $C^{2+\alpha}$ was proved by Escher and Simonett [ES]. When $n=2$, Elliot and Janovsky [EJ] showed the existence and uniqueness of weak solutions formulated by a parabolic variational inequality in a Sobolev space. (See also Gustafsson [G].) Using this variational notion of solutions, Sakai $[\mathrm{S}]$ showed that for $n=2, \Gamma_{t}(u)$ for all time $t>0$ is either analytic or has a cusp-type singularity caused by collision of two free boundary components.

In this paper we prove a quantitative, geometric version of Sakai's theorem in general dimensions. Of independent interest is an estimate on the free boundary speed, or, equivalently, the size of the spatial gradient on the free boundary (part (c) of Theorems 1.1 and 1.2).

Our first main theorem concerns global solutions starting from a Lipschitz initial free boundary. (See section 9 for the precise definition of global 
solutions.) Let $e_{n}=(0, \ldots, 0,1) \in \mathbb{R}^{n}$ and

$$
\Omega_{0}=\left\{x \in \mathbb{R}^{n}: x_{n}<g\left(x^{\prime}\right), x=\left(x^{\prime}, x_{n}\right) \in \mathbb{R}^{n-1} \times \mathbb{R}\right\}
$$

where $g$ is Lipschitz continuous with Lipschitz constant $L \leq L_{n}$, a dimensional constant. There is a positive harmonic function $u_{0}$ in $\Omega_{0}$ that vanishes on $\partial \Omega_{0}$, and it is unique up to a constant multiple. Because there is only uniqueness up to a constant multiple, we must normalize the solution at subsequent times $t$.

Theorem 1.1. (Theorem 9.2) There exists a unique, global solution $u$ of $(H S)$ in $\mathbb{R}^{n} \times[0, \infty)$ with initial positive phase $\Omega_{0}(u)=\Omega_{0}, u(x, 0)=u_{0}(x)$, normalized at infinity by

$$
\lim _{R \rightarrow \infty} \frac{u\left(-R e_{n}, t\right)}{u_{0}\left(-R e_{n}\right)}=1 \text { for each fixed } t>0 .
$$

Moreover the following properties hold.

(a) For $t>0$ the free boundary $\Gamma_{t}(u)$ is smooth and is a Lipschitz graph with respect to $e_{n}$ with Lipschitz constant $L$;

(b) $u$ is smooth in $\bar{\Omega}_{t}(u)$ for $t>0$;

(c) If $x \in \Gamma_{0}(u)$ and $x+d e_{n} \in \Gamma_{t}(u)$ with $d \geq 0$, then

$$
C^{-1} \leq \frac{\left|D u\left(x+d e_{n}, t\right)\right|}{\left|D u\left(x-d e_{n}, t\right)\right|} \leq C
$$

for a constant $C>0$ depending only on dimension.

Note that part (a) says that the Lipschitz constant is preserved (or decreases) with time. In fact, we show in Theorem 9.2(d) that if $g\left(-x^{\prime}\right)=$ $g\left(x^{\prime}\right)$, then $u\left(-x^{\prime}, x_{n}, t\right)=u\left(x^{\prime}, x_{n}, t\right)$, and the Lipschitz constant decreases to zero locally uniformly as $t \rightarrow \infty$.

$L_{n}$ can be taken to be any number $L_{n}<a_{n}$, where $a_{n}$ is the Lipschitz constant appeared in our earlier work [CJK]. For $n=2$ we have $a_{2}=1$, for which the initial harmonic function $u(x, 0)$ vanishes on the boundary at a rate bounded below by the square of the distance to the boundary. For a Lipschitz constant $L<a_{2}$, some points of the free boundary can stay fixed 
for small $t>0$, and the free boundary need not be smooth for those values of $t$. (See [JK], [KLV].)

Theorem 1.1 is proved using Theorem 1.2, our main local theorem. We also show that blow-up limits of local solutions are global solutions as in Theorem 1.1. The local theorem says, roughly speaking, that $u$ and $\Gamma_{t}(u)$ stay smooth as long as there is no invasion of fingers (another component of $\Omega(u)$ ) from the top portion of the local neighborhood.

Let $\Omega_{0}$ be, as above, the region below the graph of the Lipschitz function $g$ with Lipschitz constant $L \leq L_{n}$. Assume further that $g(0)=0$.

Theorem 1.2. (Corollary 5.6, Theorem 6.3 and Corollary 7.4) Suppose $u$ is a solution of $(H S)$ in $B_{2}(0) \times[0, T]$ with initial positive phase $\Omega_{0} \cap B_{2}(0)$, and $u$ satisfies $u\left(-e_{n}, 0\right)=1$ with

$$
\begin{gathered}
u(x, s) \leq 10 u(x, t), \quad u(x, s) \leq\left(1+A t^{-1+a}(t-s)\right) u(x, t) \text { for } 0 \leq s<t \leq T, \\
u\left(-e_{n}, t\right) \leq A u\left(-e_{n}, 0\right)=A \text { for } 0 \leq t \leq T,
\end{gathered}
$$

and

$$
\Omega_{T}(u) \cap B_{2}(0) \subset\left(\Omega_{0}+c_{0} e_{n}\right) .
$$

Then there is a constant $c_{0}>0$ depending only on dimension and on $A>0$ such that the following properties hold:

$$
\text { If } 0<r<c_{0} \text { is chosen sufficiently small that } u\left(r e_{n}, T / 2\right)>0 \text {, then }
$$

(a) The free boundary $\Gamma_{t}(u)$ is $C^{1}$ and is a Lipschitz graph with respect to $e_{n}$ with Lipschitz constant $L^{\prime}<L_{n}$ in $B_{r}(0)$, and $L^{\prime} \rightarrow L$ as $r \rightarrow 0$.

(b) The spatial normal of $\Gamma_{t}(u)$ in $B_{r}(0)$ is continuous in space and time.

(c) If $x \in \Gamma_{0}(u) \cap B_{r}(0)$ and $x+d e_{n} \in \Gamma_{t}(u) \cap B_{r}(0)$, then

$$
C^{-1} \leq \frac{\left|D u\left(x+d e_{n}, t\right)\right|}{\left|D u\left(x-d e_{n}, t\right)\right|} \leq C
$$

for a constant $C>0$ depending only on dimension.

The role of constant $A$ in Theorem 1.2 is clarified in the body of the paper, where a slightly stronger theorem is proved than is stated here. Our previous theorem in [CJK] gives smoothness for sufficiently small time, but 
depends on global features of the initial configuration. That theorem also treated only constant boundary data $f(x, t)=c$.

Concerning the validity of the hypotheses, we show in Lemma 2.14 that (1.1) is satisfied by suitably re-scaled solutions of $(H S)$ in $\left(\mathbb{R}^{n}-K\right) \times[0, \infty)$ with $u(x, t)=f(x, t), x \in K$ for a fixed boundary $K$, provided $f(x, t)$ is smooth and is strictly positive. Hypothesis (1.3) serves to define the time scale $T$ : it is chosen so that the free boundary moves at most a small multiple of unit distance in time $T$. The distance $r$ is the distance the free boundary moves in a comparable time $T / 2$. The normalization $u\left(-e_{n}, 0\right)=1$ and the second condition in (1.1) implies that $T \leq T_{0}(n, A)$. On the other hand, due to (1.2) $T$ can be chosen to be bounded below by a positive constant depending only on the dimension. Thus under conditions (1.1) and (1.2), one should think of $T$ as comparable to unit size.

Hypothesis (1.2) is the last vestige of a global hypothesis. It is valid if $T$ is sufficiently small and in many typical situations. Because it measures the change in size of $u$ at a point well inside the region, it can only fail if there is a change that swamps the effects of the initial conditions. We believe that (1.2) only goes wrong when there are collisions.

The main idea of the proof is to compare solutions at each scale to solutions on star-shaped Lipschitz domains, to which the estimates of [CJK] apply. We use the notion of viscosity solutions and the main tool is the comparison principle (Theorem 2.7). The crucial first step is a suitably localized Carleson-type estimate.

In section 2 we state several preliminary results including the definition and properties of viscosity solutions of (HS). In section 3 we prove a Carleson-type estimate (3.1). This property is used in Section 4 to show that level sets of $u$ are flat (Proposition 4.1). In Section 5 we prove the nondegeneracy of the solution on the free boundary and the Lipschitz continuity of $\Gamma(u)$. In Section 6 and 7 iteration methods developed in [CJK] are used to derive further spatial regularity of the solution and the free boundary. The key steps in the iteration process are estimates on the change of direction of $D u$ over time (Lemma 6.1 and Lemma 7.1). In Section 8 we prove regularity of $\Gamma(u)$ in time. In Section 9, as an application of the main results obtained in previous sections, we prove existence, uniqueness and regularity properties of global solutions of (HS) with Lipschitz initial free boundary with appropriate conditions at infinity. At the same time, we show that the blow-up limits of local solutions are global solutions of the type in Theorem 1.1 . 


\section{Preliminary results}

We will recall the definition of viscosity solutions for (HS) introduced in [K1]. Roughly speaking, viscosity sub and supersolutions are defined by comparison with local (smooth) super and subsolutions. In particular, classical solutions of (HS) are also viscosity sub and supersolutions.

Let $K$ be a compact subset of $\mathbb{R}^{n}$ and $Q=\left(\mathbb{R}^{n}-K\right) \times(0, \infty)$. Also let $\Sigma$ be a cylindrical domain $D \times(a, b) \subset \mathbb{R}^{n} \times \mathbb{R}$, where $D$ is an open subset of $\mathbb{R}^{n}$.

For a nonnegative real valued function $u(x, t)$ defined in a cylindrical domain $D \times(a, b)$,

$$
\begin{aligned}
& \Omega(u)=\{(x, t): u(x, t)>0\}, \quad \Omega_{t}(u)=\{x: u(x, t)>0\}, \\
& \Gamma(u)=\partial\{(x, t): u(x, t)=0\}, \quad \Gamma_{t}(u)=\partial\{x: u(x, t)=0\} .
\end{aligned}
$$

Definition 2.1. A nonnegative upper semi-continuous function u defined in $\Sigma$ is a viscosity subsolution of (HS) if

(a) for each $a<T<b$ the set $\overline{\Omega(u)} \cap\{t \leq T\}$ is bounded; and

(b) for every $\phi \in C^{2,1}(\Sigma)$ such that $u-\phi$ has a local maximum in $\overline{\Omega(u)} \cap$ $\left\{t \leq t_{0}\right\} \cap \Sigma$ at $\left(x_{0}, t_{0}\right)$,

(i) $\quad-\Delta \phi\left(x_{0}, t_{0}\right) \leq 0 \quad$ if $u\left(x_{0}, t_{0}\right)>0$.

(ii) $\quad\left(\phi_{t}-|D \phi|^{2}\right)\left(x_{0}, t_{0}\right) \leq 0 \quad$ if $\left(x_{0}, t_{0}\right) \in \Gamma(u)$ if $-\Delta \phi\left(x_{0}, t_{0}\right)>0$.

Note that because $u$ is only lower semi-continuous there may be points of $\Gamma(u)$ at which $u$ is positive.

Definition 2.2. A nonnegative lower semi-continuous function $v$ defined in $\Sigma$ is a viscosity supersolution of (HS) if for every $\phi \in C^{2,1}(\Sigma)$ such that $v-\phi$ has a local minimum in $\Sigma \cap\left\{t \leq t_{0}\right\}$ at $\left(x_{0}, t_{0}\right)$,

(i) $\quad-\Delta \phi\left(x_{0}, t_{0}\right) \geq 0 \quad$ if $v\left(x_{0}, t_{0}\right)>0$,

(ii) If $\left(z_{0}, t_{0}\right) \in \Gamma(v),|D \phi|\left(x_{0}, t_{0}\right) \neq 0$ and

$$
-\Delta \varphi\left(x_{0}, t_{0}\right)<0
$$

then

$$
\left(\phi_{t}-|D \phi|^{2}\right)\left(x_{0}, t_{0}\right) \geq 0
$$


Definition 2.3. $u$ is a viscosity subsolution of (HS) with initial data $u_{0}$ and fixed boundary data $f>0$ if

(a) $u$ is a viscosity subsolution in $\bar{Q}$,

(b) $u=u_{0}$ at $t=0 ; u \leq f$ on $\partial K$;

(c) $\overline{\Omega(u)} \cap\{t=0\}=\overline{\Omega\left(u_{0}\right)}$;

Definition 2.4. $u$ is a viscosity supersolution of (HS) with initial data $u_{0}$ and fixed boundary data $f$ if $v$ is a viscosity supersolution in $\bar{Q}$ with $v=v_{0}$ at $t=0$ and $v \geq f$ on $\partial K$.

For a nonnegative real valued function $u(x, t)$ defined in a cylindrical domain $D \times(a, b)$,

$$
u^{*}(x, t)=\limsup _{(\xi, s) \in D \times(a, b) \rightarrow(x, t)} u(\xi, s) .
$$

Definition 2.5. $u$ is a viscosity solution of (HS) (with boundary data $u_{0}$ and $f$ ) if $u$ is a viscosity supersolution and $u^{*}$ is a viscosity subsolution of (HS) (with boundary data $u_{0}$ and $f$.)

Definition 2.6. We say that a pair of functions $u_{0}, v_{0}: \bar{D} \rightarrow[0, \infty)$ are (strictly) separated (denoted by $u_{0} \prec v_{0}$ ) in $D \subset \mathbb{R}^{n}$ if

(i) the support of $u_{0}, \operatorname{supp}\left(u_{0}\right)=\overline{\left\{u_{0}>0\right\}}$ restricted in $\bar{D}$ is compact and

(ii) in $\operatorname{supp}\left(u_{0}\right) \cap \bar{D}$ the functions are strictly ordered:

$$
u_{0}(x)<v_{0}(x)
$$

The following properties of viscosity solutions are frequently used in our paper.

Theorem 2.7. (Comparison principle, [K1]) Let $u, v$ be respectively viscosity sub- and supersolutions in $D \times(0, T) \subset Q$ with initial data $u_{0} \prec v_{0}$ in $D$. If $u \leq v$ on $\partial D$ and $u<v$ on $\partial D \cap \bar{\Omega}(u)$ for $0 \leq t<T$, then $u(\cdot, t) \prec v(\cdot, t)$ in $D$ for $t \in[0, T)$.

Theorem 2.8. ([K2]) Let $u$ be a viscosity solution of (HS). Then $u$ is harmonic in $\Omega(u)$. Indeed $u(x, t)=h_{t}(x)$, where

$$
h_{t}(x)=\inf \left\{v \in \mathcal{P} \text { with } v=1 \text { on } \partial K \text { and } v \geq 0 \text { on } \Gamma_{t}\right\} .
$$

where $\mathcal{P}$ is the set of superharmonic functions in $\Omega_{t}$ which are lower semi-continuous in $\bar{\Omega}_{t}$.

Moreover $\Gamma\left(u^{*}\right)=\Gamma(u)$. 
Next we state several properties of harmonic functions:

Lemma 2.9. (Dahlberg, see [D]) Let $u_{1}, u_{2}$ be two nonnegative harmonic functions in a domain $D$ of $\mathbb{R}^{n}$ of the form

$$
D=\left\{\left(x^{\prime}, x_{n}\right) \in \mathbb{R}^{n-1} \times \mathbb{R}:\left|x^{\prime}\right|<2,\left|x_{n}\right|<2 M, x_{n}>f\left(x^{\prime}\right)\right\}
$$

with $f$ a Lipschitz function with constant less than $M$ and $f(0)=0$. Assume further that $u_{1}=u_{2}=0$ along the graph of $f$. Then for

$$
D_{1 / 2}=\left\{\left|x^{\prime}\right|<1,\left|x_{n}\right|<M, x_{n}>f\left(x^{\prime}\right)\right\}
$$

we have

$$
0<C_{1} \leq \frac{u_{1}\left(x^{\prime}, x_{n}\right)}{u_{2}\left(x^{\prime}, x_{n}\right)} \cdot \frac{u_{2}(0, M)}{u_{1}(0, M)} \leq C_{2}
$$

with $C_{1}, C_{2}$ depending only on $M$.

Lemma 2.10. (Jerison and Kenig, see [JK]) Let D, $u_{1}$ and $u_{2}$ be as in Lemma 2.9. Assume further that

$$
\frac{u_{1}(0, M / 2)}{u_{2}(0, M / 2)}=1
$$

Then, $u_{1}\left(x^{\prime}, x_{n}\right) / u_{2}\left(x^{\prime}, x_{n}\right)$ is Hölder continuous in $\bar{D}_{1 / 2}$ for some coefficient $\alpha$, both $\alpha$ and the $C^{\alpha}$ norm of $u_{1} / u_{2}$ depending only on $M$.

Lemma 2.11. (Caffarelli, see [C1]) Let $u$ be as in Lemma 2.9. Then there exists $c>0$ depending only on $M$ such that for $0<d<c \frac{\partial}{\partial x_{n}} u(0, d) \geq 0$ and

$$
C_{1} \frac{u(0, d)}{d} \leq \frac{\partial u}{\partial x_{n}}(0, d) \leq C_{2} \frac{u(0, d)}{d}
$$

where $C_{i}=C_{i}(M)$.

Lemma 2.12. (Caffarelli, see [C1]) Let $u$ be harmonic in $B_{1}$. Then there exists $\epsilon_{0}>0$ such that if

$$
u(x+\epsilon e) \geq u(x) \text { for } \epsilon>\epsilon_{0} \text { and } x, x+\epsilon e \in B_{1}(0)
$$

for a unit vector $e \in \mathbb{R}^{n}$ then $e \cdot \nabla u \geq 0$ in $B_{1 / 2}(0)$. 
Lemma 2.13. ([JeKi], Lemma 4.1) Let $\Omega$ be Lipschitz domain contained in $B_{10}(0)$. There exists a dimensional constant $\beta_{n}>0$ such that for any $\zeta \in \partial \Omega, 0<2 r<1$ and positive harmonic function $u$ in $\Omega \cap B_{2 r}(\zeta)$, if $u$ vanishes continuously on $B_{2 r}(\zeta) \cap \partial \Omega$, then for $x \in \Omega \cap B_{r}(\zeta)$,

$$
u(x) \leq C\left(\frac{|x-\zeta|}{r}\right)^{\beta_{n}} \sup \left\{u(y): y \in \partial B_{2 r}(\zeta) \cap \Omega\right\}
$$

where $C$ depends only on the Lipchitz constants of $\Omega$.

We finish this section by showing that (1.1) is a generic assumption.

Lemma 2.14. Let $w$ solve $(H S)$ in $\left(\mathbb{R}^{n}-K\right) \times[0, \infty)$ with smooth boundary data $f>0$ on $\partial K$ and initial positive phase $\Omega_{1}$. Let us normalize such that $u\left(-e_{n}, 0\right)=1$. Then (1.1) holds with

$$
T=\sup \left\{t: \sup _{x_{i} \in K, 0 \leq \tau_{i} \leq t} \frac{f\left(x_{1}, \tau_{1}\right)}{f\left(x_{2}, \tau_{2}\right)} \leq 10\right\}
$$

and with

$$
A=\sup \left\{f_{t}(x, t): x \in K, 0 \leq t \leq T\right\} .
$$

Proof. 1. Let $A$ as given above. Then for any $T>0$ we have $f(x, t) \leq$ $(1+A \epsilon) f(x, s)$ where $|t-s| \leq \epsilon$ and $0 \leq t, s \leq T$. By Theorem 2.7, for any $\epsilon>0$

$$
u\left(x, t+t_{0}\right) \leq(1+A \epsilon t) u\left(x,(1+\epsilon) t+t_{0}\right) \text { for } 0 \leq t \leq 1 / A
$$

for any $0 \leq t_{0}$. Differentiating in $\epsilon>0$ yields

$$
\left(A t u+t u_{t}\right)\left(x, t+t_{0}\right)=t\left(A_{0} u+u_{t}\right)\left(x, t+t_{0}\right)>0 .
$$

Since $t_{0}$ is arbitrary, we conclude

$$
u_{t} \geq-A u \text { for } 0 \leq t \leq T
$$

This proves the second inequality in (1.1).

2. By definition of $f, f \in[a, 10 a]$ with $a>0$ for $0 \leq t \leq T$. Since the positive phase $\Omega_{t}(u)$ expands in time, it is clear from the maximum principle of harmonic functions that $u(x, s) \leq 10 u(x, t)$ if $0 \leq s<t \leq T$. 


\section{A Carleson-type estimate}

Our first goal is to show the $\epsilon$-flat result, i.e. to show that the level sets of $u$ stays $\epsilon$-close to those of Lipschitz functions with small Lipschitz constants, at least for a small amount of time. For this we will first prove that $u$ is "almost" increasing in the direction of $-e_{n}$ in appropriate space-time scale. From this section, suppose $u$ is a solution of $(\mathrm{HS})$ in $B_{2}(0) \times[0, T]$ with initial positive phase $\Omega_{0} \cap B_{2}(0)$.

Proposition 3.1. There is a constant $c_{0}>0$ depending only on dimension and on $A>0$ such that if $u$ satisfies the first condition of (1.1), (1.2) and (1.3), and $0<r<c_{0}$ is sufficiently small that $u\left(r e_{n}, T / 2\right)>0$, then the following property holds: for small $t>0$ such that $u\left(r e_{n}, t\right)=0$,

$$
\max _{x \in \partial B_{a}(0)} u(x, t) \leq C u\left(-a e_{n}, t\right)
$$

where $a=r^{\gamma}>r, 0<\gamma<1$ and $C$ are dimensional constants.

$\diamond$ For $\Lambda \subset \mathbb{R}^{n}$, denote

$$
\Lambda+s e_{n}=\left\{x+s e_{n}: x \in \Lambda\right\} .
$$

$\diamond$ For $P \in B_{2}(0) \cap\left(\mathbb{R}^{n}-\bar{\Omega}_{0}\right)$, denote by $t(P ; u)$, the time the free boundary of $u$ reaches $P$, i.e.,

$$
t(P ; u)=\sup \{t>0: u(P, t)=0\} .
$$

The proof of Proposition 3.1 follows from the following lemma:

Lemma 3.2. There is a dimensional constant $c_{0}^{\prime}>0$ such that if $u$ satisfies (1.3) with $c_{0}^{\prime}$, and $0<r<c_{0}^{\prime}$ is sufficiently small that $u\left(r e_{n}, T\right)>0$, then the following property holds:

$$
\int_{0}^{t_{0}} \max _{x \in \partial B_{a}(0)} u(x, t) d t \leq C \int_{0}^{t_{0}} u\left(-a e_{n}, t\right) d t
$$

where $t_{0}=t\left(r e_{n} ; u\right), a=r^{\gamma}>r, \gamma<1$ and $C$ are dimensional constants. Further suppose $u$ satisfies the first condition of (1.1), and for $0 \leq t \leq t_{0}$

$$
2^{-m_{0}^{2} / 2} \leq \frac{u\left(-e_{n}, t\right)}{u\left(-e_{n}, 0\right)} \leq 2^{m_{0}^{2} / 2}
$$

where $m_{0}$ is the largest integer such that $r \leq 2^{-m_{0}}$. Then

$$
\max _{x \in \partial B_{a}(0)} u(x, t) \leq C u\left(-a e_{n}, t\right)
$$

for $0 \leq t \leq t_{0} / 2$ and a dimensional constant $C$. 
Proof of Proposition 3.1. Let $m_{0}$ be the smallest integer such that

$$
A \leq 2^{m_{0}^{2} / 2} \text { and } 2^{-m_{0} / \gamma}<c_{0}^{\prime} .
$$

Then by Lemma 3.2, (3.1) holds for $a=2^{-m_{0}}\left(r=2^{-m_{0} / \gamma}\right)$ and small $t$ such that

$$
t \leq \min \left\{t\left(r e_{n} ; u\right), T\right\} / 2
$$

and hence the result follows.

For the proof of Lemma 3.2, we first show the following two lemmas.

Lemma 3.3. If $x_{0} \in B_{2}(0)$ and $\min \left\{u(x, T): x \in B_{s}\left(x_{0}\right)\right\}=0$, then

$$
\int_{0}^{T} u\left(x_{0}, t\right) d t \leq C(n) s^{2} .
$$

Lemma 3.4. There is a dimensional constant $c_{0}^{\prime}>0$ such that if $u$ satisfies (1.3) with $c_{0}^{\prime}$, and $0<r<c_{0}^{\prime}$ is sufficiently small that $u\left(r e_{n}, T\right)>0$, then the following property holds: for $t_{0}=t\left(r e_{n} ; u\right)$,

$$
\Omega_{t_{0}} \cap B_{2^{-m}}(0) \subset\left(\Omega_{0}+2^{-5 m} e_{n}\right)
$$

where $m \geq 1$ is any integer such that $r<r^{\gamma} \leq 2^{-m}, 0<\gamma<1$ is a dimensional constant.

\section{Proof of Lemma 3.3: Let}

$$
r_{0}=\inf \left\{r: \min \left\{u(x, 0): x \in B_{r}\left(x_{0}\right)\right\}=0\right\} .
$$

and change the coordinate so that $x_{0}=0$. We construct a radially symmetric barrier as follows: let $\phi(x)$ be the harmonic function in $B_{1}(0)-B_{1 / 2}(0)$ with $\phi=0$ on $\partial B_{1}(0)$ and $\phi=1$ on $\partial B_{1 / 2}(0)$. Let

$$
\alpha(t)=C_{1} u(0, t) \text { and } r^{2}(t)=C(n) \int_{0}^{t} \alpha(s) d s+r^{2},
$$

where $C_{1}>0$ is a dimensional constant to be chosen later. Then

$$
h(x, t):=\alpha(t) \phi\left(r(t)^{-1} x\right) .
$$

is a subsolution of (HS), since

$$
V=r^{\prime}(t) \leq|D \phi|=C(n) \alpha(t) r^{-1}(t) \text { on } \Gamma_{t}(h)=\partial B_{r(t)}(0) .
$$


Now we compare $h$ with $u$ in the domain

$$
\Sigma:=\left(\mathbb{R}^{n}-B_{r(t) / 2}(0)\right) \times(0, T) .
$$

At $t=0 h \prec u$ by definition of $r$ and Harnack inequality for $u$. As long as $\Omega_{t}(h) \subset \Omega_{t}(u), h(\cdot, t) \leq u(\cdot, t)$ on $\partial B_{r(t) / 2}(0)$ due to the Harnack inequality for $u$, if $C_{1}$ is chosen sufficiently small. Therefore $h$ cannot cross $u$ from below for the first time in $\Omega(h)$. On the other hand, as long as $h(\cdot, t) \leq u(\cdot, t)$ on $\partial B_{r(t) / 2}(0), h(\cdot, t) \prec u(\cdot, t)$ due to Theorem 2.7. Thus we conclude that $h \leq u$ in $\Sigma$, and in particular

$$
B_{r(T)}(0) \subset \Omega_{T}(h) \subset \Omega_{T}(u) .
$$

By our hypothesis $r(T) \leq s$, which means

$$
\int_{0}^{T} \alpha(t) d t=\int_{0}^{T} C_{1} u(0, t) d t \leq C(n) s^{2}
$$

This yields our conclusion.

Proof of Lemma 3.4: We prove the lemma by induction for $1 \leq m \leq$ $m_{0}$, where $m_{0}$ is the largest integer such that $r^{\gamma} \leq 2^{-m_{0}}$. Suppose (3.3) holds for some $m \in \mathbb{N}$. To simplify notations, denote

$$
B_{1}=B_{2^{-m}}(0), B_{2}=B_{2^{-m} \cdot 4 / 5}(0), B_{3}=B_{2^{-m} \cdot 3 / 5}(0), B_{4}=B_{2^{-m-1}}(0),
$$

and $p_{0}=-2^{-m-1} e_{n}$.

Let $h(x, t)=h^{t}(x)$ be the harmonic function in $\left(\Omega_{0}+2^{-5 m} e_{n}\right) \cap B_{1}$ such that

$$
h^{t}(x)= \begin{cases}0 & \text { on } \Gamma_{0}+2^{-5 m} e_{n}, \\ u(x, t) & \text { on } \partial B_{1} .\end{cases}
$$

Due to the maximum principle for harmonic functions, $u(\cdot, t) \leq h^{t}$ in $B_{1}$. In particular, for $x \in \Gamma_{0} \cap B_{2}$ and $0 \leq s \leq 2^{-5 m}$,

$$
\begin{aligned}
u\left(x+s e_{n}, t\right) & \leq h^{t}\left(x+s e_{n}\right) \\
& \leq C\left(\frac{2^{-5 m}}{2^{-m}}\right)^{k} h^{t}\left(x-\frac{2^{-m}}{10} e_{n}\right) \\
& \leq C 2^{-4 k m} 2^{m} \int_{\partial B_{1}} u(x, t) d x \\
& \leq C 2^{-4 k m}\left(u\left(p_{0}, t\right)+2^{m} \int_{S} u(x, t) d x\right)
\end{aligned}
$$


where $S=\partial B_{1} \cap\left\{\Gamma_{0}+s e_{n}: 0 \leq s \leq 2^{-5 m}\right\}, C$ and $k$ are positive dimensional constants. Denote the last term of the above inequality by $m_{t}$, i.e.,

$$
m_{t}=C 2^{-4 k m}\left(u\left(p_{0}, t\right)+2^{m} \int_{S} u(x, t) d x\right) .
$$

It then follows from above arguments that

$$
u\left(x+s e_{n}, t\right) \leq m_{t}
$$

for $x \in \Gamma_{0} \cap B_{2}$ and $0 \leq s \leq 2^{-5 m}$. Next, let $w_{t}$ be the harmonic function in $\Omega_{0} \cap B_{2}$ such that

$$
w_{t}(x)= \begin{cases}u(x, t) & \text { on } \partial B_{2} \cap \Omega_{0}, \\ m_{t} & \text { on } \Gamma_{0} \cap B_{2} .\end{cases}
$$

Then $u(x, t) \leq w_{t}(x)$ in $\Omega_{0} \cap B_{2}$, and by Lemma 2.9

$$
\begin{aligned}
\max _{x \in \Omega_{0} \cap \partial B_{3}} u(x, t) & \leq \max _{x \in \partial B_{3}} w_{t}(x) \\
& \leq C\left(m_{t}+u\left(p_{0}, t\right)\right) .
\end{aligned}
$$

Above inequality and (3.4) imply

$$
\begin{aligned}
\max _{x \in \partial B_{3}} u(x, t) & \leq C\left(m_{t}+u\left(p_{0}, t\right)\right) \\
& \leq C\left(2^{-4 k m+m} \int_{S} u(x, t) d x+u\left(p_{0}, t\right)\right) .
\end{aligned}
$$

Now for $x_{0} \in \Gamma_{0} \cap B_{4}$, construct a wedge $\Lambda_{x_{0}}$ with vertex $x_{0}$ :

$$
\Lambda_{x_{0}}=x_{0}+\left\{\left(x^{\prime}, x_{n}\right): L\left|x^{\prime}\right|<x_{n}<\frac{2^{-m}}{10}\right\},
$$

which is contained in $B_{3}-\Omega_{0}$. Let $v(x, t)$ solve (HS) with

$$
\left\{\begin{array}{l}
v(x, t)=\max _{x \in \partial B_{3}} u(x, t) \quad \text { on the fixed boundary } \partial B_{3}, \\
\Gamma_{0}(v)=\Lambda_{x_{0}}
\end{array}\right.
$$

Note that by Theorem $2.7 u \leq v$ for $0 \leq t \leq t_{0}$. Hence for

$$
\bar{d}\left(x_{0}\right):=\max \left\{s: u\left(x_{0}+s e_{n}, t_{0}\right)>0, x_{0}+s e_{n} \in B_{4}\right\},
$$


we get

$$
\begin{aligned}
\bar{d}\left(x_{0}\right) & \leq d\left(x_{0}, t_{0} ; v\right):=\inf \left\{d: x_{0}+d e_{n} \in \Gamma_{t_{0}}(v)\right\} \\
& \leq C\left(\int_{0}^{t_{0}} \frac{\max _{x \in \partial B_{3}} u(x, t)}{2^{-m-1}} d t\right)^{\alpha} \\
& \leq C\left(2^{-4 k m+2 m} \int_{0}^{t_{0}} \int_{S} u(x, t) d x d t+\int_{0}^{t_{0}} \frac{u\left(p_{0}, t\right)}{2^{-m}} d t\right)^{\alpha}
\end{aligned}
$$

where

$$
S=\partial B_{1} \cap\left\{\Gamma_{0}+s e_{n}: 0 \leq s \leq 2^{-5 m}\right\},
$$

the second inequality follows from Lemma 2.13 with a constant $1 / 2<\alpha<1$ depending on $n$, and the last inequality follows from (3.5).

On the other hand, if (3.3) holds for $m$, then Lemma 3.3 implies

$$
\int_{0}^{t_{0}} u(x, t) d t \leq C 2^{-10 m} \text { for } x \in S .
$$

It follows that

$$
\int_{0}^{t_{0}} \int_{S} u(x, t) d x d t \leq C 2^{-15 m}
$$

We proceed to find an upper bound of $\int_{0}^{t_{0}} \frac{u\left(p_{0}, t\right)}{2^{-m}} d t$ : construct a wedge

$$
\tilde{\Lambda}_{x_{0}}=x_{0}+\left\{\left(x^{\prime}, x_{n}\right):-\frac{2^{-m}}{10}<x_{n}<-L\left|x^{\prime}\right|\right\}
$$

in $\Omega_{0}$, and a ball $\tilde{B}$ inside $\tilde{\Lambda}_{x_{0}}$. Let $w$ solve (HS) with

$$
\left\{\begin{array}{l}
w(x, t)=c u\left(p_{0}, t\right) \quad \text { on the fixed boundary } \partial \tilde{B} \\
\Gamma_{0}(w)=\tilde{\Lambda}_{x_{0}} .
\end{array}\right.
$$

If $c$ is a sufficiently small dimensional constant, then due to Harnack inequality for harmonic functions and Theorem 2.7

$$
w \leq u \text { for } 0 \leq t \leq t_{0} .
$$

Due to Lemma 2.13

$$
\left(\int_{0}^{t_{0}} \frac{c u\left(p_{0}, t\right)}{2^{-m}} d t\right)^{\beta} \leq d\left(0, t_{0} ; u\right):=\inf \left\{d: d e_{n} \in \Gamma_{t_{0}}(u)\right\}=r
$$


for a dimensional constant $\beta>1$. Let $\gamma=1 /(12 \beta)$ and let $m_{0}$ be the largest integer such that $r^{\gamma} \leq 2^{-m_{0}}$. Then if $m<m_{0}$, (3.6) and (3.7) imply

$$
\begin{aligned}
\bar{d}\left(x_{0}\right) & \leq C\left(2^{-4 k m-13 m}+r^{1 / \beta} 2^{m}\right)^{\alpha} \\
& \leq C\left(2^{-13 m}+2^{-11 m}\right)^{1 / 2} \\
& \leq C 2^{-11 m / 2} .
\end{aligned}
$$

Here $C$ is a dimensional constant, and the second inequality follows from $r^{\gamma}=r^{1 /(12 \beta)}<2^{-m_{0}}<2^{-m}$. Thus if $m>m_{1}=m_{1}(n)$, then

$$
\bar{d}\left(x_{0}\right) \leq 2^{-5(m+1)} .
$$

For $m \leq m_{1},(3.3)$ holds if we let $c_{0}^{\prime}<2^{-5 m_{1}}$.

We are now ready to prove Lemma 3.2 using Lemma 3.4.

Proof of Lemma 3.2: Let $m_{0}$ be the largest integer such that $r^{\gamma} \leq$ $2^{-m_{0}}$, where $\gamma$ is given as in Lemma 3.4. For $0 \leq t \leq t_{0}=t\left(r e_{n} ; u\right)$ and $1 \leq m \leq m_{0}$, denote

$$
A_{t, m}=\max \left\{u(x, t): x \in \partial B_{2^{-m}}(0) \cap\left\{\Gamma_{0}+s e_{n}: 0 \leq s \leq 2^{-5 m}\right\}\right\} .
$$

Note that, due to (3.5),

$$
\begin{aligned}
A_{t, m+1} & \leq \max _{\partial B_{2}-m \cdot 3 / 5} u(x) \\
& \leq C\left(2^{m} \int_{\partial B_{2}-m}(0) \cap\left\{\Gamma_{0}+s e_{n}: 0 \leq s \leq 2^{-5 m}\right\}\right. \\
& \leq C\left(2^{-4 m} A_{t, m}+u\left(p_{m}, t\right)\right)
\end{aligned}
$$

where $p_{m}=-2^{-m-1} e_{n}$ and $C$ is a dimensional constant. Then by iteration on $m$ we obtain that

$$
A_{t, m_{0}} \leq C^{m_{0}} 2^{-2 m_{0}^{2}} A_{t, 1}+\sum_{m=1}^{m_{0}} C^{m_{0}-m_{2}} 2^{-2\left(m_{0}^{2}-m^{2}\right)} u\left(p_{m}, t\right) .
$$

Here, by Harnack inequality, $u\left(p_{m}, t\right)$ is bounded as follows:

$$
u\left(p_{m}, t\right) \leq C_{0}^{m_{0}-m} u\left(p_{m_{0}}, t\right)
$$

where $C_{0}$ is a constant depending on $n$. Hence, if $m_{0} \geq \bar{m}$ for a sufficiently large constant $\bar{m}$ depending on $n$ and $C_{0}$, then

$$
A_{t, m_{0}} \leq 2^{-m_{0}^{2}}\left(A_{t, 1}+u\left(p_{m_{0}}, t\right)\right)
$$


where $p_{m_{0}}=-2^{-m_{0}-1} e_{n}$. Observe that

$$
\int_{0}^{t_{0}} A_{t, 1} d t \leq \int_{0}^{t_{0}} \max _{x \in B_{1 / 2}(0)} u(x, t) d t \leq C \int_{\partial B_{1}(0)} \int_{0}^{t_{0}} u(x, t) d t d x \leq C
$$

where the last inequality follows from Lemma 3.3.

Next let

$$
a=2^{-m_{0}-1} \approx r^{\gamma} .
$$

Then by (3.9) and (3.10)

$$
\begin{aligned}
\int_{0}^{t_{0}} \max _{x \in \partial B_{a}(0)} u(x, t) d t & \leq 2^{-m_{0}^{2}} \int_{0}^{t_{0}} A_{t, 1} d t+C \int_{0}^{t_{0}} u\left(-a e_{n}, t\right) d t \\
& \leq C\left(2^{-m_{0}^{2}}+\int_{0}^{t_{0}} u\left(-a e_{n}, t\right) d t\right)
\end{aligned}
$$

It follows that

$$
\begin{aligned}
2^{\left(-m_{0}-1\right) / \gamma} & \leq r \\
& \leq C\left(\int_{0}^{t_{0}} \frac{\max _{x \in \partial B_{a}(0)} u(x, t)}{a} d t\right)^{\alpha} \\
& \leq C\left(2^{-m_{0}^{2}+m_{0}}+2^{m_{0}} \int_{0}^{t_{0}} u\left(-a e_{n}, t\right) d t\right)^{\alpha} \\
& \leq C 2^{\left(-m_{0}^{2}+m_{0}\right) \alpha}+C\left(2^{m_{0}} \int_{0}^{t_{0}} u\left(-a e_{n}, t\right) d t\right)^{\alpha}
\end{aligned}
$$

where the second inequality follows from Lemma 2.13 with $1 / 2<\alpha<1$ depending on $n$, and the third inequality follows from (3.11) and (3.12). If $m_{0} \geq \bar{m}(n)>>1$,

$$
C 2^{\left(-m_{0}^{2}+m_{0}\right) \alpha+1} \leq 2^{\left(-m_{0}-1\right) / \gamma}
$$

where $C>1$ is a constant given in (3.13). Thus (3.13) implies

$$
2^{\left(-m_{0}-1\right) / \gamma} \leq C\left(2^{m_{0}} \int_{0}^{t_{0}} u\left(-a e_{n}, t\right) d t\right)^{\alpha}
$$

and we get

$$
2^{\left(-m_{0}^{2}+m_{0}\right) \alpha} \leq 2^{\left(-m_{0}-1\right) / \gamma} \leq C\left(2^{m_{0}} \int_{0}^{t_{0}} u\left(-a e_{n}, t\right) d t\right)^{\alpha} .
$$


By (3.12) and (3.14)

$$
\begin{aligned}
\int_{0}^{t_{0}} \max _{x \in \partial B_{a}(0)} u(x, t) d t & \leq C\left(2^{-m_{0}^{2}}+\int_{0}^{t_{0}} u\left(-a e_{n}, t\right) d t\right) \\
& \leq C \int_{0}^{t_{0}} u\left(-a e_{n}, t\right) d t .
\end{aligned}
$$

To prove the second part of the lemma, we further assume $u(x, s) \leq$ $10 u(x, t)$ for $0 \leq s<t \leq T$, and

$$
1 / 10 \leq \frac{u\left(-e_{n}, t\right)}{u\left(-e_{n}, 0\right)} \leq \tilde{M}:=2^{m_{0}^{2} / 2}
$$

for $0 \leq t \leq t_{0}=t\left(r e_{n} ; u\right)$. Then by Harnack inequality for harmonic functions

$$
\max _{0 \leq t \leq t_{0}} u\left(-a e_{n}, t\right) \leq 10 u\left(-a e_{n}, t_{0}\right) \leq C^{m_{0}} \tilde{M}
$$

where $a=2^{-m_{0}-1}$ and $C$ is a dimensional constant. Hence by Lemma 2.13

$$
\left(\int_{0}^{t_{0}} \frac{C^{m_{0}} \tilde{M}}{a} d t\right)^{\beta} \geq r
$$

for a constant $0<\beta<1$ depending on $n$, which implies

$$
\frac{1}{t_{0}} \leq C^{m_{0}} 2^{m_{0}} r^{-1 / \beta} \tilde{M} \leq 2^{k m_{0}} \tilde{M}
$$

where $k$ is a positive dimensional constant.

Since

$$
10 A_{s, 1} \geq A_{t_{0} / 2,1} \text { for } s \geq t_{0} / 2, \text { and } \int_{0}^{t_{0}} A_{t, 1} d t \leq C,
$$

it follows that

$$
\frac{t_{0}}{20} A_{t_{0} / 2,1} \leq \int_{t_{0} / 2}^{t_{0}} A_{t, 1} d t \leq C .
$$

Hence if $t \leq t_{0} / 2$, then

$$
A_{t, 1} \leq 10 A_{t_{0} / 2,1} \leq C \frac{1}{t_{0}} \leq C 2^{k m_{0}} \tilde{M}
$$

for positive dimensional constants $C$ and $k$. Then by (3.9)

$$
A_{t, m_{0}} \leq C 2^{-m_{0}^{2}}\left(2^{k m_{0}} \tilde{M}+u\left(p_{m_{0}}, t\right)\right)
$$


and, due to Harnack inequality,

$$
u\left(p_{m_{0}}, t\right) \geq C 2^{-\tilde{k} m_{0}} u\left(-e_{n} / 2, t\right) \geq C 2^{-\tilde{k} m_{0}}
$$

for $\tilde{k}=\tilde{k}(n)>0$. Since $\tilde{M}=2^{m_{0}^{2} / 2}$, if $m_{0} \geq \bar{m}$ for a sufficiently large $\bar{m}$ depending on $n$, then (3.15) implies

$$
A_{t, m_{0}} \leq C u\left(p_{m_{0}}, t\right)
$$

and hence for $a=2^{-m_{0}-1}$

$$
\max _{x \in \partial B_{a}(0)} u(x, t) \leq C u\left(-a e_{n}, t\right) .
$$

\section{$4 \quad \epsilon$-flatness of level sets of $u$ in a ball}

In this section, we show the flatness property of $u$ satisfying the hypothesis of Proposition 3.1, i.e., satisfying the first condition of (1.1), (1.2) and (1.3). $0<r<c_{0}$ will denote a sufficiently small constant such that $u\left(r e_{n}, T / 2\right)>$ 0 .

To show the $\epsilon$-flatness of $u$ (Proposition 4.1), we will construct a starshaped barrier $w$ for $u$. Proof of the proposition will need more arguments than that of Lemma 3.2 in [CJK] due to the fact that the Hölder continuity of $u$ in time (Corollary 2.3 in [CJK]) does not hold here.

We begin by introducing some notations.

$\diamond$ For nonzero vectors $v_{1}, v_{2}$ in $\mathbb{R}^{n}$, we define $\alpha\left(v_{1}, v_{2}\right)$ to be the (smaller) angle between $v_{1}$ and $v_{2}$.

$\diamond$ We say that a function $f$ has a cone of monotonicity

$$
W(\theta, \nu):=\left\{p \in \mathbb{R}^{n}: \alpha(p, \nu)<\theta\right\}
$$

or $f$ is monotone for the cone $W(\theta, \nu)$ in $D \subset \mathbb{R}^{n}$ if $f$ is monotone increasing along every direction $p \in W(\theta, \nu)$ in $D$.

Consider a star-shaped domain $\Omega^{\prime}$ such that

(i) $\Omega^{\prime} \cap B_{2}(0)=\Omega_{0} \cap B_{2}(0)$;

(ii) $\Omega^{\prime}$ is star-shaped with respect to every $x \in K^{\prime} \subset \Omega^{\prime}$ for a sufficiently large ball $K^{\prime}$. 
Let $v_{0}$ be the harmonic function in $\Omega^{\prime}-K^{\prime}$ with data 1 on $\partial K^{\prime}$ and 0 on $\partial \Omega^{\prime}$, and let $v$ be the solution of (HS) with $v(x, 0)=v_{0}(x)$ and $v(x, t)=1$ on $\partial K^{\prime}$. It follows from comparison arguments (see Lemma 3.3 in [CJK]) that $v$ is starshaped with respect to $K^{\prime}$. In particular, $v(\cdot, t)$ is monotone in $B_{1}(0)$ for the cone $W\left(\theta-\epsilon,-e_{n}\right)$ for $0 \leq t \leq t\left(e_{n} ; v\right)$, if $K^{\prime}$ is sufficiently large depending on $\epsilon$. Here $\epsilon$ is a small constant to be chosen later.

Based on $v$, we will construct a supersolution $w^{\text {sup }}$ and a subsolution $w^{s u b}$ of (HS) such that in $B_{r}(0), w^{s u b} \leq u \leq w^{s u p}$ and the level sets of $w^{s u b}$ and $w^{\text {sup }}$ are close to each other.

We begin by constructing a concentric ball $B_{2}$ of $B_{1}(0)$ as follows: Let $k_{0}, k_{1}$ and $k_{2}$ be sufficiently large numbers satisfying

$$
k_{0} \gg k_{1}, \quad \gamma=\frac{k_{0}}{k_{0}+k_{1}+k_{2}}
$$

where $\gamma$ is a constant given as in Lemma 3.4. ( $k_{0}, k_{1}$ and $k_{2}$ will be determined later in the proof.) Let $k=k_{0}+k_{1}+k_{2}$ and let $\epsilon=r^{1 / k}$ $\left(\epsilon^{k}=r\right)$. We denote

$$
B_{2}:=B_{\epsilon^{k_{0}}}(0) \subset B_{1}(0)=: B_{1} .
$$

Since $r^{\gamma}=\epsilon^{k_{0}}$, Lemma 3.4 implies

$$
\Omega_{t_{0}} \cap B_{2} \subset\left(\Omega_{0}+\epsilon^{5 k_{0}} e_{n}\right) \cap B_{2}
$$

for $t_{0}:=t\left(r e_{n} ; u\right)$. Next define

$$
H=\left(\Gamma_{0}-\epsilon^{k_{0}+k_{1}} e_{n}\right) \cap \frac{2}{3} B_{2}
$$

where $\frac{2}{3} B_{2}=B_{2 \epsilon^{k_{0} / 3}}(0)$. Observe that

$$
\operatorname{radius}\left(B_{2}\right) \gg \operatorname{dist}\left(H, \Gamma_{0}\right) \gg \max _{x \in \Gamma_{t_{0}} \cap B_{2}} \operatorname{dist}\left(\mathrm{x}, \Gamma_{0}\right) .
$$

If $k_{1}$ is sufficiently large, Lemma 2.10 implies that for any positive harmonic functions $h$ and $g$ in $B_{2} \cap \Omega_{0}$, vanishing on $\Gamma_{0}$, there exists a constant $c>0$ such that

$$
1-\epsilon \leq \frac{h}{c g} \leq 1+\epsilon \text { on } H
$$

Let $h(x, t):=h^{t}(x)$ be the harmonic function in $B_{2} \cap \Omega_{0}$ such that

$$
h^{t}= \begin{cases}0 & \text { on } \Gamma_{0}, \\ u(x, t) & \text { on } \partial B_{2} \cap \Omega_{0} .\end{cases}
$$


Then

$$
h^{t}(x) \leq u(x, t) \text { in } B_{2} .
$$

Since $h:=h^{t}$ and $g:=v_{0}$ are both positive harmonic functions in $B_{2} \cap \Omega_{0}$, which vanish on $\Gamma_{0}, h$ and $g$ satisfies (4.1) with $c=c_{t}$, i.e., there is a constant $c_{t}$ such that

$$
1-\epsilon \leq \frac{h^{t}}{c_{t} v_{0}} \leq 1+\epsilon \text { on } H \text {. }
$$

Hence we get

$$
(1-\epsilon) c_{t} v_{0} \leq u(x, t) \text { for } x \in H .
$$

Similarly,let $\tilde{h}(x, t):=\tilde{h}^{t}(x)$ be a harmonic function in $B_{2} \cap\left(\Omega_{0}+\epsilon^{5 k_{0}} e_{n}\right)$ such that

$$
\tilde{h}^{t}= \begin{cases}0 & \text { on } \Gamma_{0}+\epsilon^{5 k_{0}} e_{n}, \\ u(x, t) & \text { on } \partial B_{2} \cap\left(\Omega_{0}+\epsilon^{5 k_{0}} e_{n}\right) .\end{cases}
$$

Then

$$
u(x, t) \leq \tilde{h}^{t}(x) \text { in } B_{2} .
$$

Since $\epsilon^{5 k_{0}} \ll \epsilon^{k_{0}+k_{1}}=\operatorname{dist}\left(\mathrm{H}, \Gamma_{0}\right)$, from a similar argument as for $h^{t}$, we obtain a constant $\tilde{c}_{t}$ such that

$$
1-\epsilon \leq \frac{\tilde{h}^{t}}{\tilde{c}_{t} v_{0}} \leq 1+\epsilon \text { on } H .
$$

Therefore we have

$$
u(x, t) \leq \tilde{h}^{t}(x) \leq(1+\epsilon) \tilde{c}_{t} v_{0}(x) \text { for } x \in H .
$$

Let $w$ be the star-shaped solution of (HS) with

$$
\left\{\begin{array}{l}
\Omega_{0}(w)=\Omega_{0}(v), \\
w(x, t)=(1-3 \epsilon) c_{t} \text { on } \partial K^{\prime} .
\end{array}\right.
$$

In other words,

$$
w(x, t)=(1-3 \epsilon) c_{t} v\left(x,(1-3 \epsilon) \int_{0}^{t} c_{s} d s\right) .
$$

We now state our main proposition: 
Proposition 4.1. Suppose $u$ satisfies the first condition of (1.1), (1.2) and (1.3) with $c_{0}>0$ depending only on dimension and on $A>0$. Suppose $0<r<c_{0}$ is sufficiently small that $u\left(r e_{n}, T / 2\right)>0$, then the following property holds: there is an integer $k>0$ depending on dimension such that if $0<s \leq r$ and $\epsilon$ satisfies $\epsilon^{k}=s$, then the level sets of $u(\cdot, t)$ stay $s \epsilon$-close to those of $w$ in $B_{2 s}(0)$ for $0 \leq t \leq t\left(s e_{n} ; u\right)$. In particular $u$ is se-monotone for the cone $W\left(\theta-\epsilon,-e_{n}\right)$ in $B_{2 s}(0)$ for $0 \leq t \leq t\left(s e_{n} ; u\right)$.

Proof. Without loss of generality, we may assume $s=r$. We will perturb $w$ by order of $\epsilon$ to construct $w^{s u p}$ and $w^{s u b}$.

Let $\tilde{w}$ be the star-shaped solution of (HS) with

$$
\left\{\begin{array}{l}
\Omega_{0}(\tilde{w})=\Omega_{0}(v), \\
\tilde{w}(x, t)=(1+3 \epsilon) \tilde{c}_{t} \text { on } \partial K^{\prime},
\end{array}\right.
$$

namely

$$
\tilde{w}(x, t)=(1+3 \epsilon) \tilde{c}_{t} v\left(x,(1+3 \epsilon) \int_{0}^{t} \tilde{c}_{s} d s\right) .
$$

Since $r=\epsilon^{k} \ll \epsilon^{k_{0}+k_{1}}$, Corollary 2.3 in [CJK] implies

$$
v\left(x, t_{0}^{\prime}\right) \leq(1+\epsilon) v_{0}(x) \text { for } x \in H
$$

where $t_{0}^{\prime}:=t\left(r e_{n} ; v\right)=t\left(\epsilon^{k} e_{n} ; v\right)$. Thus for $0 \leq s \leq r, x \in H$ and $t=$ $t\left(s e_{n} ; w\right)$,

$$
\begin{aligned}
w(x, t) & =(1-3 \epsilon) c_{t} v\left(x, t\left(s e_{n} ; v\right)\right) \\
& \leq(1-2 \epsilon) c_{t} v_{0}(x) \\
& \leq(1-\epsilon) u(x, t)
\end{aligned}
$$

where the last inequality follows from (4.4). Similarly if $x \in H$ and $t=$ $t\left(s e_{n} ; \tilde{w}\right)$ then

$$
\begin{aligned}
\tilde{w}(x, t) & =(1+3 \epsilon) \tilde{c}_{t} v\left(x, t\left(s e_{n} ; v\right)\right) \\
& \geq(1+3 \epsilon) \tilde{c}_{t} v_{0}(x) \\
& \geq(1+\epsilon) u(x, t)
\end{aligned}
$$

where the last inequality follows from (4.8).

Based on $\tilde{w}$ we construct a supersolution $w^{\text {sup }}$ as in Lemma 3.2 of [CJK], by bending the free boundaries of $\tilde{w}$ up above $\Gamma_{t}(u)$ in $\frac{2}{3} B_{2}-\frac{1}{3} B_{2}$. Define

$$
\psi(x)=\frac{1}{|x|^{2}} \cdot\left(x_{1}, \ldots, x_{n-1},-x_{n}+2|x|^{2}\right)
$$


where $x=\left(x_{1}, \ldots, x_{n}\right) \in \mathbb{R}^{n}$. Since $\psi$ is the composition of reflection about $x_{n}=1$ and the conformal mapping sending $x$ to $\frac{x}{|x|^{2}}$,

$$
\phi:=\psi\left(x+e_{n}\right)-e_{n}
$$

is also conformal. Observe that $\phi$ fixes 0 and will bend the free boundary of $\tilde{w}$ up above $\Gamma_{t}(u)$ in $\frac{2}{3} B_{2}-\frac{1}{3} B_{2}$. Set $S_{t}$ to be the strip between $H$ and $\Gamma_{t}(\tilde{w})$ and define

$$
w^{s u p}(x, t)=(1+\epsilon) \tilde{w}\left(\phi^{-1}(x), t\right) \text { in } R:=\phi\left(S_{t}\right) \cap \frac{2}{3} B_{2} .
$$

Note that $w^{\sup }(\cdot, t)$ is harmonic in $R$ and

$$
\Gamma_{t}\left(w^{\text {sup }}\right)=\phi\left(\Gamma_{t}(\tilde{w})\right)
$$

in $\frac{2}{3} B_{2}$. By a similar argument as in Lemma 3.2 of [CJK], $w^{\text {sup }}$ is a supersolution in $\frac{2}{3} B_{2} \times[0, T]$.

Now we compare $w^{\text {sup }}$ and $u$ in

$$
\Sigma:=\left(\frac{1}{2} B_{2} \cap R\right) \times[0, \tilde{T}],
$$

where $\tilde{T}=\min \left\{t_{0}, t\left(r e_{n} ; w^{\text {sup }}\right)\right\}, t_{0}=t\left(r e_{n} ; u\right)$.

First note that, due to (4.6), (4.10) and the fact

$$
\max _{\phi^{-1}(R)}|\phi(x)-x| \approx \epsilon^{2 k_{0}} \ll \epsilon^{k_{0}+k_{1}} \approx \operatorname{dist}\left(H, \Gamma_{0}(u)\right),
$$

we have

$$
u(x, t) \leq w^{\text {sup }}(x, t) \text { on } \phi(H) \cap \frac{1}{2} B_{2} \times[0, \tilde{T}] .
$$

Secondly observe that in $\frac{2}{3} B_{2}-\frac{1}{3} B_{2}$ and for $0 \leq t \leq \tilde{T}$

$$
\Gamma(u) \subset\left(\bigcup_{s \leq \epsilon^{5 k_{0}}}\left(\Gamma_{0}(u)+s e_{n}\right)\right) \times[0, \tilde{T}]
$$

due to Lemma 3.4. On the other hand,

$$
\Gamma_{t}\left(w^{s u p}\right)=\phi\left(\Gamma_{t}(\tilde{w})\right) \subset \bigcup_{s \geq \epsilon^{2 k_{0}}}\left(\Gamma_{0}(\tilde{w})+s e_{n}\right)=\bigcup_{s \geq \epsilon^{2 k_{0}}}\left(\Gamma_{0}(u)+s e_{n}\right) .
$$

Hence it follows that

$$
\Omega(u)+\epsilon^{2 k_{0}} / 2 e_{n} \subset \Omega\left(w^{\text {sup }}\right) \text { in }\left(\frac{2}{3} B_{2}-\frac{1}{3} B_{2}\right) \times[0, \tilde{T}]
$$


Due to (4.10), (4.12) and Lemma 2.10, we obtain

$$
u(x, t) \leq w^{\text {sup }}(x, t) \text { on } \partial\left(\frac{1}{2} B_{2} \cap R\right) \times[0, \tilde{T}] .
$$

Therefore $u \leq w^{\text {sup }}$ on the parabolic boundary of $\Sigma$, and Theorem 2.7 yields that

$$
u \leq w^{\text {sup }} \text { in } \Sigma .
$$

Similarly we construct a subsolution $w^{\text {sub }}$ as follows. Let $\bar{\psi}$ be the composition of reflection about $x_{n}=1$ and the conformal mapping sending $x$ to $\frac{x}{\left|x-2 e_{n}\right|^{2}}$, then $\bar{\phi}:=\bar{\psi}\left(x+e_{n}\right)-e_{n}$ will bend the free boundary of $w$ down below $\Gamma_{t}(u)$ in $\frac{2}{3} B_{2}-\frac{1}{3} B_{2}$. Let us define

$$
w^{s u b}(x, t)=(1-\epsilon) w\left(\bar{\phi}^{-1}(x), t\right)
$$

Then $w^{s u b}(\cdot, t)$ is harmonic in $\bar{R}:=\frac{2}{3} B_{2} \cap \bar{\phi}\left(\bar{S}_{t}\right)$, where $\bar{S}_{t}$ is defined similarly as in $S_{t}$. From an argument parallel to the one for showing (4.13), we get

$$
w^{s u b} \leq u \in\left(\frac{1}{2} B_{2} \cap \bar{R}\right) \times\left[0, T^{\prime}\right]
$$

where $T^{\prime}=\min \left\{t_{0}, t\left(r e_{n} ; w^{s u b}\right)\right\}$.

Let $B_{3}=B_{2 r}(0)=B_{2 \epsilon^{k}}(0)$. Then in $B_{3}$, if $0 \leq t \leq T^{\prime}$ and $0<s \leq r$, the level sets $\left\{x \in B_{3}: u(x, t)=s\right\}$ are located between $\left\{x \in B_{3}: w^{\text {sup }}(x, t)=\right.$ $s\}$ and $\left\{x \in B_{3}: w^{s u b}(x, t)=s\right\}$, which are in the $\epsilon r$-neighborhood of $\left\{x \in B_{3}: \tilde{w}(x, t)=s\right\}$ and $\left\{x \in B_{3}: w(x, t)=s\right\}$, respectively. Since $w$ is monotone in $B_{1}(0)$ for the cone $W\left(\theta-\epsilon,-e_{n}\right)$, to conclude it suffices to prove that the level sets $\left\{x \in B_{3}: w(x, t)=s\right\}$ are contained in the $r \epsilon$-neighborhood of the level sets $\left\{x \in B_{3}: \tilde{w}(x, t)=s\right\}$ of $\tilde{w}$.

Recall that $w$ and $\tilde{w}$ are star-shaped solutions with the same initial domain, and with different fixed boundary values $(1-\epsilon) c_{t}$ and $(1+\epsilon) \tilde{c}_{t}$, respectively. By (4.4) and (4.8), $c_{t}$ and $\tilde{c}_{t}$ satisfy

$$
1 \leq \frac{\tilde{c_{t}}}{c_{t}} \leq(1+2 \epsilon) \frac{\tilde{h}^{t}(x)}{h^{t}(x)}
$$

for $x \in H$. Let $x_{0}=-\epsilon^{k_{0}+k_{1}} e_{n} \in H$, then

$$
\tilde{h}^{t}\left(x_{0}\right) \leq\left(1+C \frac{\max _{x \in \partial B_{2}} u(x, t)}{u\left(-\epsilon^{k_{0}} e_{n}, t\right)} \epsilon^{\beta k_{0}}\right) h^{t}\left(x_{0}\right)
$$


for $C>0$ and $\beta>0$ depending on $n$, since $h_{t}=\tilde{h}_{t}=u(x, t)$ on $\partial B_{2} \cap\left\{h_{t}>\right.$ $0\}$, and since

$$
\Gamma_{t}(\tilde{h})=\Gamma_{t}(h)+\epsilon^{5 k_{0}} e_{n} \text { in } B_{2},
$$

with $\epsilon^{5 k_{0}}$ much smaller than $\epsilon^{k_{0}+k_{1}}=d\left(x_{0}, \Gamma_{t}(h)\right)$ for $0 \leq t \leq T^{\prime}$. Therefore if $k_{0}$ is sufficiently large, (4.14) implies

$$
1 \leq \frac{\tilde{c_{t}}}{c_{t}} \leq 1+\frac{\max _{x \in \partial B_{2}} u(x, t)}{u\left(-\epsilon^{k_{0}} e_{n}, t\right)} \epsilon .
$$

By Proposition 3.1,

$$
\max _{x \in \partial B_{2}} u(x, t) \leq C u\left(-\epsilon^{k_{0}} e_{n}, t\right)
$$

and thus

$$
1 \leq \frac{\tilde{c_{t}}}{c_{t}} \leq 1+C \epsilon
$$

for a dimensional constant $C$. Now (4.16) and Lemma 3.3 of [CJK] yields that the level sets of $w$ and $\tilde{w}$ are contained in the $C r \epsilon$-neighborhood of each other.

\section{Non-degeneracy and Lipschitz continuity}

Our next goal is to improve Proposition 4.1 to conclude that $u(\cdot, t)$ is Lipschitz in space in $B_{r}(0)$ for $0 \leq t \leq t\left(r e_{n} ; u\right)$. For this purpose we first need to show that $u$ is non-degenerate on $\Gamma(u)$ in $\epsilon$-scale (Proposition 5.1).

Proposition 5.1. Let $u$ be a solution of $(H S)$ with $0 \in \Gamma$ in $B_{2}(0) \times \mathbb{R}^{+}$. In addition suppose that $t\left(e_{n} ; u\right)=1$, $u$ satisfies (1.1) with $A \leq 1$ and $u(\cdot, t)$ is $\epsilon$-monotone for $0<\epsilon<\epsilon_{n}$ for the cone $W\left(\theta,-e_{n}\right), \theta>\pi / 4$ in the region $B_{2}\left(e_{n}\right)$ for $t \in[0,1]$, where $\epsilon_{n}$ is a dimensional constant and $t_{0}=t\left(e_{n}\right)$. Then there exists a dimensional $C>0$ such that

$$
B_{1 / 2}\left(e_{n}\right) \cap\left(\Omega_{t}+s e_{n}\right) \subset \Omega_{(1+C \epsilon) t}
$$

for $t\left(e_{n} / 2 ; u\right) \leq t \leq t\left(e_{n} ; u\right)$ and $0 \leq s \leq \epsilon$.

The proof is parallel to that of Proposition 4.1 in [CJK], but we state the proof below to illustrate the use of (1.1). 
Proof. For $P_{1} \in B_{1 / 2}\left(e_{n}\right) \cap \Omega_{t}(u)$, denote $t_{1}=t\left(P_{1}\right)$. The goal is to show that $u\left(P_{1}+s e_{n},(1+C \epsilon) t_{1}\right)>0$ for some $C(n)>0$. Let $P_{2}=P_{1}+\frac{1}{5} e_{n}, R=$ $B_{1 / 2}\left(P_{2}\right)-B_{1 / 10}\left(P_{2}\right)$ and $\Sigma=R \times\left[0, t_{1}\right]$. Define the function

$$
w(x, t):=\inf _{B_{\epsilon \varphi(x)}(x)} u\left(y-\epsilon e_{n}, t\right)
$$

where $\varphi$ defined in $R$ satisfies the following properties:

$$
\begin{array}{ll}
\text { (a) } \quad \Delta\left(\varphi^{-Q_{n}}\right)=0 & \text { in } R ; \\
\text { (b) } \varphi=A_{n} & \text { on } \partial B_{1 / 10}\left(P_{2}\right) ; \\
\text { (c) } \varphi=1 / \sqrt{2} & \text { in } \partial B_{1 / 2}\left(P_{2}\right) .
\end{array}
$$

Here $Q_{n}>0$ is a dimensional constant chosen such that Lemma 9 in [C1] applies, $A_{n}$ is chosen sufficiently large that $\varphi\left(P_{1}\right)>2$. Note that also $|D \varphi| \leq C$ where $C$ depends on $Q_{n}$ and $A_{n}$.

Now we compare $w$ and $u$ in $\Sigma$. First due to the $\epsilon$-monotonicity of $u$ (in space) we can argue as in [CJK] to check that $w \geq u$ on the parabolic boundary of $\Sigma$. Observe that, formally speaking, $w$ satisfies

$$
\frac{w_{t}}{|D w|} \geq(1-O(\epsilon))|D w| \text { on } \Gamma(w) \cap \Sigma,
$$

due to the definition of $w$. It follows that for some constant $C=C(n)>0$

$$
w_{1}(x, t):=(1+C \epsilon) w(x,(1+C \epsilon) t) \text { is a supersolution of }(\mathrm{HS}) \text { in } \Sigma,
$$

(For rigorous proof see the proof of Proposition 4.1 in [CJK].)

Note that $t_{1} \leq 1$. Thus if $u$ satisfies (1.1) with $A<1$ then $w \leq w_{1}(x, t)$ and therefore Theorem 2.7 yields

$$
u(x, t) \leq w_{1}(x, t) \text { in } \Sigma .
$$

On the other hand by (c) and the definition of $w, w(x, t) \leq u\left(x+s e_{n}, t\right)$ at $P$ for $0 \leq s \leq \epsilon$ and thus we can conclude.

The following corollary and its proof correspond to Corollary 4.4 in [CJK]. 
Corollary 5.2. Let $u, \epsilon$ be as given in Proposition 5.1 and let $t=t\left(e_{n} ; u\right)$. Then there exists $C(n)>0$ such that for $x \in \Gamma_{t}(u) \cap B_{1 / 2}\left(e_{n}\right)$,

$$
\sup _{y \in B_{2 \epsilon}(x)} u_{1}(y, t) \geq C \epsilon
$$

In terms of our original $u$, the following holds:

Corollary 5.3. Let $P_{0} \in \Gamma_{t_{0}}(u)$ and $d\left(P_{0}, P_{1}\right)=r, t_{1}=t\left(P_{1} ; u\right)>t_{0}$. Furthermore suppose that $u$ is $r \epsilon$-monotone for $0<\epsilon<\epsilon_{n}$ for the cone $W\left(\theta, e_{n}\right), \theta>\pi / 4$ in $B_{2 r}\left(P_{0}\right) \times\left[t_{0}, t_{1}\right]$. Then there exits a dimensional constant $C>0$ such that

$$
\sup _{y \in B_{2 r \epsilon}(x)} u(y, t) \geq \frac{C}{t_{1}-t_{0}} r \epsilon .
$$

for $t=t_{1}$.

Remark. Corollary 5.3 states that the lower bound on the normal velocity, or $|D u|$ on $\Gamma(u)$, is proportional to the distance $d$ it has moved in $e_{n}$ direction from its initial position. Furthermore this lower bound is obtained uniformly in the space neighborhood of size $d$, suggesting that the regularization of the free boundary occurs in scale of $d$ in space and $t\left(d e_{n} ; u\right)$ in time.

Proof. Let

$$
\tilde{u}(x)=\frac{\left(t_{1}-t_{0}\right)}{r} u\left(r x,\left(t_{1}-t_{0}\right) t+t_{0}\right)
$$

and apply Corollary 5.2 to $\tilde{u}$.

The following lemma is a modification of Theorem 5.1 in [CJK]. The proof is parallel to that in [CJK].

Lemma 5.4. Suppose $u$ solves $(H S)$ in $B_{3}(0) \times\left[0, t_{0}\right], t_{0}=t\left(e_{n} ; u\right)$ such that

(a) $u(\cdot, t)$ is $\epsilon$-monotone for a cone $W\left(\theta,-e_{n}\right)$ in $B_{2}\left(e_{n}\right)$ for $0 \leq t \leq t_{0}$.

(b) u satisfies

$$
\sup _{y \in B_{2 \epsilon}(x)} u(y, t) \geq C \epsilon
$$

for $x \in \Gamma_{t}(u)$ for $0 \leq t \leq t_{0}$. 
If $\pi / 2-\theta<\tan ^{-1}\left(L_{n}\right)$ for a dimensional constant $L_{n}>0$ and $0<\epsilon<1 / 100$ then there is a constant $0<\lambda<1$ depending only on $C$ and $n$ such that $u(\cdot, t)$ is $\lambda \epsilon$-monotone for $W\left(\theta^{\prime},-e_{n}\right)$ in $B_{2-\epsilon^{1 / 4}}\left(e_{n}\right)$ for $\epsilon^{1 / 6} \leq t \leq t_{0}$ with $\theta^{\prime}=\theta-\epsilon^{1 / 14}$.

Corollary 5.5. Let $u$ be as in Proposition 5.1. Then $\Gamma_{t}(u)$ is Lipschitz in space with Lipschitz constant $L<L^{\prime}=L+C \epsilon^{1 / 14}<L_{n}$ in $B_{2}(0) \times[1 / 2,1]$, where $C$ is a dimensional constant.

Proof. 1. Due to Corollary 5.3, (5.2) holds for $u$ in $B_{2}(0) \times[1 / 4,1]$ with dimensional constant $C$. By iterating Lemma 5.4 and Proposition 5.1, it follows that $u(\cdot, t)$ is monotone in the cone $W\left(\theta^{\prime},-e_{n}\right)$ in $B_{s}\left(e_{n}\right)$ for $b \leq t \leq$ 1 , where

$$
s=2-C \epsilon^{1 / 4} \Sigma_{k=0}^{\infty} \lambda^{k / 4}, \quad b=c+C \epsilon^{1 / 6} \Sigma_{k=0}^{\infty} \lambda^{k / 6}
$$

for $C=C(n, A)$, a dimensional constant $0<c<1$, and

$$
\theta^{\prime}=\theta-\epsilon^{1 / 14} \Sigma_{k=0}^{\infty} \lambda^{k / 14} .
$$

Hence if $a(r, A)$ is chosen sufficiently small such that the corresponding $\epsilon$ in Proposition 4.1 is sufficiently small, the lemma holds for $1 / 2 \leq t \leq 1$.

Corollary 5.6. Let $u, r$ and $k$ as given in Proposition 4.1. Then for $0<$ $s<r, u$ is Lipschitz in space with Lipschitz constant $L<L^{\prime}+s^{(14 k)^{-1}}<L_{n}$ in $B_{s}(0) \times\left[0, t\left(s e_{n} ; u\right)\right]$.

Proof. For any $0<a \leq r$ and $x_{0} \in \Gamma_{0} \cap B_{2}(0)$, let

$$
\tilde{u}(x, t)=\frac{t(a)}{a} u\left(a\left(x-x_{0}\right), t(r) t\right), t(r)=t\left(x_{0}+a e_{n} ; u\right) .
$$

We then apply Proposition 4.1 and Corollary 5.5 with $\tilde{u}$ to derive the lemma for $\frac{1}{2} t_{0} \leq t \leq t_{0}$. Since $r$ is arbitrary small, we get the Lipschitz property of $\Gamma_{t}(u)$ in $B_{a}(0)$ for $0<t<t\left(a e_{n} ; u\right)$.

Corollary 5.7. Let $u$ and $r$ be as given in Proposition 4.1. Then for $0 \leq$ $t \leq t\left(s e_{n} ; u\right)$ with $0 \leq s \leq r$,

$$
1 / C_{1} \leq \frac{u\left(-s e_{n}, t\right)}{u\left(-s e_{n}, 0\right)} \leq C_{1}
$$

and

$$
\frac{s^{2}}{C_{1} u\left(-s e_{n}, 0\right)} \leq t\left(s e_{n} ; u\right) \leq C_{1} \frac{s^{2}}{u\left(-s e_{n}, 0\right)},
$$

where $C_{1}=C_{1}(n, A)$. 
Proof. Due to Corollary $5.6 u$ is Lipschitz in space in $B_{r}(0) \times\left[0, t\left(r e_{n} ; u\right)\right]$ with $r=r(n, A)$. Hence it follows that $\Gamma_{t}(u)$ is Lipschitz in $B_{r}(0)$ for $0<t<t\left(r e_{n} ; u\right)$, and which implies

$$
\max _{\partial B_{r}(0)} u(x, t) \leq C u\left(-r e_{n}, t\right)
$$

where $0<t<T$ and $C$ is a dimensional constant. Furthermore, since $u\left(-e_{n}, t\right) \leq A u\left(-e_{n}, 0\right)$, By Harnack inequality

$$
\max _{\partial B_{s}(0)} u(x, t) \leq C_{1} u\left(-s e_{n}, 0\right)
$$

for $0<s<r, 0<t<t\left(s e_{n} ; u\right)$ and $C_{1}=C_{1}(n, A)$. Then the results follow from a similar argument as in Theorem 2.1 and Corollary 2.2 of [CJK].

\section{Regularity in space}

In section 6-7 we modify iteration argument used in section 9-10 of [CJK] to yield further regularity of $\Gamma(u)$.

The main idea is that the nice properties of $u$ in the positive phase propagates to the free boundary over time. In particular in [CJK] $u_{t} \leq$ $C|D u|^{2}$ in the positive phase, due to the fact that $\Omega_{t}(u)$ was a globally Lipschitz domain with Lipschitz constant $L<L_{n}$. This property, ensures that the direction of the spatial gradient $D u\left(-e_{n}, t\right)$, which represents the direction of propagation of the flow in unit scale, does not change too quickly in time (see Lemma 9.1 in [CJK]).

In our case $u_{t}$ is no longer bounded since $\Omega_{t}(u)$ may not be Lipschitz outside of $B_{1}(0)$. Hence we need a new argument to control the change of $\frac{D u}{|D u|}$ over time.

Lemma 6.1. Let $u$ solve $(H S)$ in $B_{1}(0) \times(-1,1)$ with (1.1), $0 \in \Gamma_{0}(u)$ and $t\left(e_{n} ; u\right)=1$. Suppose

(a) $u(\cdot, t)$ is monotone for the cone $W\left(e_{n}, \theta_{0}\right)$ in $B_{1}(0)$, with $\theta_{0}>\pi / 2-$ $\tan ^{-1}\left(L_{n}\right)$.

(b) $u(\cdot, t)$ is monotone for the cone $W\left(\nu_{l}, \theta_{l}\right)$ in $B_{2^{-l+1}}(0) \times\left(0,2^{-l} \delta_{l}\right)$ with $\delta_{l}=\pi / 2-\theta_{l}>l^{-2}$.

Then for integer $l>l_{0}$ where $l_{0}$ depending only on $\theta_{0}$ and $n$, there exist a unit vector $\nu_{l+1} \in \mathbb{R}^{n}$ and $0<h_{0}(n)<1$ and $0<r_{0}(n)<1$ such that 
$u(\cdot, t)$ is monotone increasing in $B_{2^{-l-5}}\left(-2^{-l-1} e_{n}\right) \times\left(0, r_{0} 2^{-l} \delta_{l}\right)$ for the cone $\eta \in W\left(\nu_{l+1}, \theta_{l+1}\right)$ with

$$
\delta_{l+1} \leq h_{0} \delta_{l}
$$

Proof. 1. Let $u$ and $w$ be given as in Proposition 4.1. Then the following is true due to Proposition 4.1:

$$
|u-w|(x, t) \leq C 2^{-\alpha l} w\left(-2^{-l} e_{n}, t\right) \text { in } B_{2^{-l+1}}(0) \times\left[0, t\left(2^{-l} e_{n} ; u\right)\right]
$$

where $C>0$ and $0<\alpha<1$ only depend on $n$.

Due to the hypothesis and $(6.1), w_{2}(\cdot, t)$ is $2^{(-\alpha-1) l}$-monotone for the cone $W\left(e_{n}, \theta_{l}\right)$ in $B_{2^{-l+1}}(0) \times\left[0, t\left(2^{-l} e_{n} ; w\right)\right]$, and thus due to Corollary 5.2 in $[\mathrm{CJK}], w(\cdot, t)$ is monotone for the cone $W\left(\nu_{l+1}, \theta_{l}^{\prime}\right)$ with

$$
\theta_{l}^{\prime}=\theta_{l}-C 2^{-\alpha / 14 l}
$$

where $C$ only depends on $\theta_{0}, n$. Let $\delta_{l}^{\prime}=\pi / 2-\theta_{l}^{\prime}$. Observe that, due to (1.1),

$$
t\left(r e_{n} ; u\right) \geq r t\left(e_{n} ; u\right)=r / 2 .
$$

In particular

$$
t\left(2^{-l} e_{n} ; w\right) \geq\left(1-2^{-\alpha l} t\left(2^{-l} e_{n} ; u\right) \geq 2^{-l-1} .\right.
$$

Note that due to Lemma 9.1 in [CJK] applied to $w$, there exist a unit vector $\nu_{l} \in \mathbb{R}^{n}, 0<r_{1}(n)<1$ and $0<h_{0}=h_{0}(n)<1$ such that

$$
\alpha\left(D w_{2}, \nu_{l}\right) \leq h_{0} \delta_{l}^{\prime} \text { in } B_{2^{-l-3}}\left(-2^{-l} e_{n}\right) \times\left[0, r_{1} 2^{-l} \delta_{l}\right] .
$$

Since $\delta_{l}>l^{-2}, h_{0} \delta_{l}^{\prime}<\tilde{h}_{0} \delta_{l}$ for sufficiently large $l$, where

$$
0<\tilde{h}_{0}=1 / 2+h_{0} / 2<1 .
$$

2. Take a unit vector $p \in \mathbb{R}^{n}$ such that

$$
\alpha\left(p, \nu_{l}\right) \leq \pi / 2-\left(2-\tilde{h}_{0}\right) \delta_{l} .
$$

Due to (6.2), for $0<\epsilon<1$ and $0 \leq t \leq r_{0} 2^{-l} \delta_{l}$, there exists $c_{0}>0$ depending on $n$ such that

$$
w\left(\cdot+2^{-l} \epsilon p, t\right) \geq c_{0} 2^{-l} \epsilon\left(1-\tilde{h}_{0}\right) \delta_{l} \frac{w\left(-2^{l} e_{n}, t\right)}{2^{-l}}+w(\cdot, t)
$$


in $B_{2^{-l-3}}\left(-2^{-l} e_{n}\right)$ for $0 \leq t \leq r_{1} 2^{-l} \delta_{l}$. Now by (6.1),

$$
\begin{aligned}
u\left(\cdot+2^{-l} \epsilon p, t\right) & \geq\left(1-C 2^{-\alpha l}\right) w\left(\cdot+2^{-l} \epsilon p, t\right) \\
& \geq\left(c_{0}\left(1-\tilde{h}_{0}\right) \epsilon \delta_{l}-C 2^{-\alpha l}\right) w\left(-2^{l} e_{n}, t\right)+w(\cdot, t) \\
& \geq\left(1-2^{-\alpha l}\right)\left(c_{0} \tilde{h}_{0} \epsilon \delta_{l}-2 C 2^{-\alpha l}\right) u\left(-2^{-l} e_{n}, t\right)+u(\cdot, t),
\end{aligned}
$$

in $B_{2^{-l-3}}\left(-2^{-l} e_{n}\right)$ for $0 \leq t \leq r_{1} 2^{-l} \delta_{l}$, where the second inequality is due to (6.3). In other words $u(\cdot, t)$ is $2^{-l} \epsilon$-monotone in the direction of $p$ in $B_{2^{-l-3}}\left(-2^{-l} e_{n}\right)$ if $l$ is sufficiently large with respect to $1-\tilde{h}_{0}, \alpha$ and $\epsilon$ such that

$$
c_{0}\left(1-\tilde{h}_{0}\right) \epsilon \delta_{l}>c_{0}\left(1-h_{0}\right) \epsilon l^{-2}>C 2^{-\alpha l} .
$$

Thus if we choose $\epsilon=\epsilon\left(\theta_{0}, n\right)>0$ small enough, then Lemma 1 in [C2] implies that

$$
p \cdot D u(x, t) \geq 0 \text { in } B_{2^{-l-5}}\left(-2^{-l} e_{n}\right) \times\left[0, r_{1} 2^{-l} \delta_{l}\right],
$$

for $l>l_{0}\left(\theta_{0}, n\right)$, which proves our assertion.

Below we state a modified version of Lemma 9.3 in [CJK]. The proof is parallel to that in [CJK]. Combined with Lemma 6.1, the following lemma says that, for $\left(x_{0}, t_{0}\right) \in \Gamma(u), \frac{D u}{D u}$ converges as we take smaller neighborhoods of $\left(x_{0}, t_{0}\right)$. The rate of this convergence, in comparison with the size of the neighborhood, determines the regularity of $\Gamma(u)$ in space.

Lemma 6.2. Let $u$ solve $(H S)$ in $B_{1}(0) \times[-\tau, \tau], 0<\tau<1$ with

$$
(0,0) \in \Gamma(u), t\left(e_{n} ; u\right)=1 \text { and }|D u|>m_{0} \text { on } \Gamma(u) .
$$

In addition suppose there exists a unit vector $\nu \in \mathbb{R}^{n}$ and $0<b_{0}<1$ such that

$$
\alpha\left(D u,-e_{n}\right) \leq \delta \text { in } B_{1}(0) \times(-\tau, \tau) .
$$

with $\delta$ smaller than a dimensional constant, and

$$
\alpha(D u(x, t), \nu) \leq b_{0} \delta \text { in } B_{1 / 16}\left(-e_{n}\right) \times(-\tau, \tau) .
$$

Then there exists a unit vector $\nu_{1} \in \mathbb{R}^{n}$ and a constant $0<c<1$ depending only on $n, m_{0}$ and $b_{0}$ such that

$$
\alpha\left(D u(x, t), \nu_{1}\right) \leq \delta_{1} \text { in } B_{1 / 2}(0) \times(-\tau / 2, \tau / 2)
$$

where $\delta_{1} \leq \delta-c \delta \tau$. 
Now we go back to our original solution $u$. Let us fix $P_{0} \in \Gamma_{0} \cap B_{r}(0)$ $s_{0} \in\left[t\left(P_{0}+s e_{n}\right), t\left(P_{0}+2 s e_{n}\right)\right], 0<s \leq r$ and $x_{0} \in \Gamma_{s_{0}}(u) \cap B_{3 s}\left(P_{0}\right)$.

Change the coordinate and re-scale as follows:

$$
\breve{u}(x, t)=\frac{\alpha}{s} u_{2}\left(s x+x_{0}, \alpha t+s_{0}\right), \alpha=t\left(s e_{n} ; u\right) .
$$

Next, we construct blow-up family $\left\{u_{l}\right\}$ of $u$ which satisfy the hypothesis of Lemma 6.1 as follows:

$$
u_{l+1}(x, t):=4^{l} \alpha_{l} \breve{u}\left(2^{-l} x, \alpha_{l} t\right),
$$

where $\alpha_{0}=1$ and for $l \geq 1$

$$
\alpha_{l}=t\left(2^{-l} e_{n} ; \breve{u}\right) .
$$

Note that $u_{l}(l=1,2 \ldots)$ is a viscosity solutions of $(\mathrm{HS})$ in $B_{1}(0) \times(-1,1)$ with the property $t\left(e_{n} ; u_{l+1}\right)=1$. Also recall that $\Gamma(u)$ is Lipschitz with Lipschitz constant $L<L_{n}$ and by Corollary 5.3

$$
\left|D u_{l}(x, t)\right| \geq m_{0} \text { for }(x, t) \in\left(B_{1}(0) \cap \Omega_{t}\left(u_{l}\right)\right) \times(-1 / 2,1) .
$$

with $m_{0}=m_{0}(n)$.

Now we apply an iteration argument, starting with sufficiently large $l$ if necessary. Suppose that $u_{l}(\cdot, t)$ is monotone for the cone $W\left(\nu_{l}, \theta_{l}\right)$ in $B_{2}(0) \times\left(-\delta_{l}, \delta_{l}\right)$ where

$$
\delta_{l}=\pi / 2-\theta_{l}>l^{-2} .
$$

Then $\breve{u}$ satisfies the hypothesis of Lemma 6.1, and thus there exists a unit vector $\nu$ and $0<r_{0}(n), h_{0}(n)<1$ such that $u_{l+1}(\cdot, t)$ is monotone increasing in $B_{1 / 16}\left(-e_{n}\right) \times\left(-2 r_{0} \delta_{l}, 2 r_{0} \delta_{l}\right)$ for the cone $W\left(\theta_{l+1}, \nu\right)$ with $\delta_{l+1} \leq h_{0} \delta_{l}$. Now Lemma 6.2 applies to $u_{l+1}$ to yield the enlarged cone of monotonicity $W\left(\nu_{l+1}, \theta_{l+1}\right)$ with

$$
\pi / 2-\theta_{l+1}=\delta_{l+1}=\delta_{l}-c r_{0} \delta_{l}^{2}, \quad c=c(n)
$$

for $u_{l+1}$ in $B_{1 / 2}(0) \times\left[-r_{0} \delta_{l}, r_{0} \delta_{l}\right]$. Now we can repeat the process with $\delta_{l+k}$, where $k=k(n)$ such that $2^{-k}<r_{0}$.

From (6.5) we obtain

$$
\delta_{l} \approx \frac{C}{l},
$$

which yields the differentiability of $\Gamma(u)$ in space at $(0,0)$ : 
Theorem 6.3. Let $u$ and $r$ be as given in Proposition 4.1, and let $\tau=$ $t\left(r e_{n} ; u\right)$. Then $\Gamma(u)$ is $C^{1}$ in space in $B_{r}(0) \times[-\tau, \tau]$. In particular, there exist constants $l_{0}, C_{0}>0$ depending only on $n$ such $\left(x_{0}, t_{0}\right) \in \Gamma(u)$,

$$
\Gamma_{t}(u) \cap B_{2^{-l}}\left(x_{0}\right) \text { for }\left|t-t_{0}\right| \leq 2^{-l}
$$

is a Lipschitz graph with respect to direction $\frac{D u}{|D u|}\left(-2^{-l} e_{n}, t_{0}\right)$, with Lipschitz constant less then $\frac{C_{0}}{l}$ if $l \geq l_{0}$.

\section{Upper bound of the spatial gradient on the free boundary}

To derive an upper bound of $|D u|$, we need stronger regularity than $C^{1}$ of $\Gamma(u)$ in space. For this we need a refined version of Lemma 6.1 as below:

Lemma 7.1. Suppose $u$ is as given in Lemma 6.1 with $l^{-2} \leq \delta_{l} \leq l^{-1}$. Then for $l \in \mathbb{N}, l>l_{0}$ where $l_{0}$ depending only on $n$ and $\theta_{0}$, there exist a unit vector $\nu_{l+1} \in \mathbb{R}^{n}$ and $0<r_{0}, h_{0}, k<1$ such that $u(\cdot, t)$ is monotone increasing in

$$
B_{2^{-l-5} / \sqrt{l}}\left(-\frac{2^{-l}}{\sqrt{l}} e_{n}\right) \times\left(0, r_{0} 2^{-l} l^{k} \delta_{l}\right)
$$

along every direction $\eta \in W\left(\theta_{1}, \nu_{1}\right)$ with

$$
\delta_{l+1} \leq h_{0} \delta_{l}
$$

Here $r_{0}=r_{0}\left(\theta_{0}, n\right)$ and $k, h_{0}$ only depends on $\theta_{0}$ and $n$.

Proof. Proceeding as in the proof of Lemma 6.1, one can construct a starshaped solution $w$ of (HS) with $s=\frac{2^{-l}}{\sqrt{l}}$. Moreover arguing as before, $w(\cdot, t)$ is monotone for the cone $W\left(e_{n}, \theta_{l}^{\prime}\right)$ in

$$
B_{2^{-l+1}}(0) \times\left[0, t\left(2^{-l} e_{n} ; w\right)\right],
$$

where $\theta_{l}^{\prime}=\theta_{l}-C 2^{-\frac{\alpha}{14} l}, C=C(n)$. Observe that $\delta_{l}^{\prime}=\delta_{l}+C 2^{-\frac{\alpha}{14} l} \leq 2 l^{-l}$ for sufficiently small $l$, and by Proposition 10.5 in [CJK] applied to $w$

$$
w_{t}(x, t)=|D w|^{2}\left(-\left(2^{l} \sqrt{l}\right)^{-1} e_{n}, t\right)\left(1+O\left(l^{-k}\right)\right)
$$

in the region

$$
B_{\frac{1}{8}\left(2^{l} \sqrt{l}\right)^{-1}}\left(-\left(2^{l} \sqrt{l}\right)^{-1} e_{n}\right) \times\left[0, t\left(2^{-l} e_{n} ; w\right)\right]
$$


where $0<k<1 / 2$ and $C>0$ depends only on $n$.

Due to Lemma 9.1 in [CJK] applied to $w$, there exists a unit vector $\nu_{l} \in \mathbb{R}^{n}$ and $0<h_{0}<1$ such that

$$
\alpha\left(D w, \nu_{l}\right) \leq h_{0} \delta_{l} \text { in } B_{\frac{1}{8}\left(2^{l} \sqrt{l}\right)^{-1}}\left(-\left(2^{l} \sqrt{l}\right)^{-1} e_{n}\right) \times\left[0, r_{0} 2^{-l} l^{k} \delta_{l}\right] .
$$

Parallel argument as in the proof of Lemma 5.1 yields the conclusion.

We can now apply an iteration argument as in section 6 to the family of functions

$$
\tilde{u}^{l+1}(x, t)=4^{l} l \beta_{l} \breve{u}\left(\frac{x}{2^{l} \sqrt{l}}, \beta_{l} t\right)
$$

where $\breve{u}$ is defined in (6.4) and

$$
\beta_{l}=t\left(\frac{2^{-l}}{\sqrt{l}} e_{n} ; u\right)
$$

Namely, $\left\{\tilde{u}^{l}\right\}$ is defined similarly as $\left\{u^{l}\right\}$ in section 6 , but replacing the scaling factor $2^{l}$ by $2^{l} \sqrt{l}$. Then the corresponding $\delta_{l}$ obtained for $\tilde{u}_{l}$ satisfies

$$
\delta_{l+1}=\delta_{l}-\delta_{l}^{2} l^{k}
$$

and we obtain $\delta_{l} \sim l^{-\gamma}$, where $1<\gamma<2$. Thus the following theorem is obtained for $\breve{u}$ :

Theorem 7.2. There exist constants $l_{0}>0$ and $1<\gamma<2$ depending only on $n$ such that for a free boundary point $\left(x_{0}, t_{0}\right) \in \Gamma(\tilde{u}) \cap\left(B_{1}(0) \times[0,1]\right)$, if $l>l_{0}$ then $\Gamma(\breve{u}) \cap B_{2^{-l}}\left(x_{0}, t_{0}\right)$ is a Lipschitz graph with Lipschitz constant less then $l^{-\gamma}$.

Then by Theorem 2.4 in [W] we obtain the upper bound of $|D \breve{u}|$ :

Corollary 7.3. The spatial gradient $D \breve{u}(\cdot, t)$ exists in $\bar{\Omega}_{t}(\breve{u}) \cap B_{1}(0)$ for $0 \leq t \leq 1$ and

$$
|D \breve{u}(\cdot, t)| \leq C \text { in } \Omega_{t}(u) \cap B_{1}(0) \text { for } 0 \leq t \leq 1,
$$

where $C$ is a dimensional constant.

In terms of our original function $u$, we obtain the following statement:

Corollary 7.4. Let $u, r$ and $\tau$ as given in Theorem 6.3. Then for any $P_{0} \in \Gamma_{0} \cap B_{r}(0), 0<t_{0}=t\left(P_{0}+s e_{n}\right)<\tau$ we have

$$
C_{1} \leq \frac{|D u(\cdot, t)|}{\left|D u\left(P_{0}-s e_{n}, t\right)\right|} \leq C_{2} \text { in } \Omega_{t}(u) \cap B_{s}\left(P_{0}+s e_{n}\right) \text { for } \frac{t_{0}}{2} \leq t \leq t_{0} .
$$

Here $C_{1}$ and $C_{2}$ are dimensional constants. 


\section{Regularity in time}

It remains to address the regularity of $\Gamma(u)$ in time. Here we will use the fact that, due to the second condition in (1.1), $e^{A t} u$ is increasing in time, and therefore one-sided limits $u\left(x, t^{-}\right)$and $u\left(x, t^{+}\right)$exists for all $(x, t)$.

Proposition 8.1. Let $u, r$ and $\tau$ be as in Theorem 6.3. Then $\Gamma(u)$ is Lipschitz continuous in time in $B_{r}(0) \times(0, \tau)$. More precisely, if $\left(x_{0}, t_{0}\right) \in$ $\Gamma(u)$, then

$$
V_{\left(x_{0}, t_{0}\right)}^{-}:=\lim _{(y, s) \in \Omega(u) \rightarrow\left(x_{0}, t_{0}\right), s<t_{0}} \frac{u_{t}}{|D u|}(y, s)=|D u|\left(x, t_{0}^{-}\right)
$$

and

$$
V_{\left(x_{0}, t_{l}\right)}^{+}:=\lim _{(y, s) \in \Omega(u) \rightarrow\left(x_{0}, t_{0}\right), s>t_{0}} \frac{u_{t}}{|D u|}(y, s)=|D u|\left(x, t_{0}^{+}\right)
$$

Remark Note that $u$ may have jump discontinuities in time, and thus one cannot ensure that free boundary velocity is continuous in time.

Proof. 1. For $l=1,2, \ldots$ let us define

$$
u^{l}(x, t)=2^{l} u\left(x_{0}+2^{-l} x, t_{0}+2^{-l} t\right) \text { in } B_{2^{l}}(0) \times\left[-2^{l}, 2^{l}\right] .
$$

Without loss of generality we may assume that $e_{n}:=\frac{D u}{|D u|}\left(x_{0}, t_{0}\right)$. Theorem 6.3 yields that

$$
\alpha\left(D u^{l}, e_{k}\right) \rightarrow 0 \text { as } l \rightarrow \infty \text { if } k \neq n,
$$

locally uniformly in $\mathbb{R}^{n}$.

Moreover, due to Theorem 7.2 and Theorem 2.4 in [W],

$$
\left|D u(\cdot, t)-D u\left(x_{0}-2^{-m} e_{n}, t\right)\right| \leq 1 / m \text { in } \Omega_{t}(u) \cap B_{2^{-m}}\left(x_{0}\right)
$$

for $\left|t-t_{0}\right| \leq 2^{-m}$.

2. Let us we define, for a given domain $\Sigma \in \mathbb{R}^{n+1}$,

$$
d_{\Sigma}(x, t):=\text { signed distance function to } \Sigma .
$$

Formally speaking,

$$
\frac{u_{t}}{|D u|}=|D u| \text { on } \Gamma(u)
$$


in a small neighborhood of $\left(x_{0}, t_{0}\right)$. In particular $d_{\Omega\left(u^{l}\right)}$ is uniformly Lipschitz in time with respect to $l$. Due to a barrier argument based on Corollary 7.3, one can show that $\Gamma\left(u^{l}\right)$ has bounded normal velocity. Therefore if we define

$$
u^{\infty}(x, t):=\limsup _{l \rightarrow \infty,(y, s) \rightarrow(x, t)} u^{l}(y, s)
$$

and

$$
u_{\infty}(x, t):=\liminf _{l \rightarrow \infty,(y, s) \rightarrow(x, t)} u^{l}(y, s),
$$

Then due to Arzeli-Ascola, $d_{\Omega\left(u^{\infty}\right)}$ and $d_{\Omega\left(u_{\infty}\right)}$ are given by a local uniform limit of respective subsequences of $\left\{d_{\Omega\left(u^{l}\right)}\right\}_{l \in \mathbb{N}}$.

3. Due to (8.1) and (8.2),

$$
u^{\infty}(x, t)=a_{1}(t)\left(a_{2}(t)-x_{n}\right)_{+}, \text {and } u_{\infty}(x, t)=b_{1}(t)\left(b_{2}(t)-x_{n}\right)_{+} .
$$

where $a_{2}(t)$ and $b_{2}(t)$ is increasing, $a_{2}(0)=b_{2}(0)=0$ and Lipschitz continuous in time. Also observe that, due to (1.1) $\left(1+2^{-l} A t\right) u^{l}$ increases in time, and thus $u^{\infty}$ and $u_{\infty}$ increases in time, and in particular $a_{1}(t)$ and $b_{1}(t)$ has one-sided limits.

4. We prove that $a_{1}(0-)=b_{1}(0-)$. Suppose

$$
\left|a_{1}(0-)-b_{1}(0-)\right|>\delta>0 .
$$

By definition of $a_{1}(t), b_{1}(t)$, one can choose sufficiently large $l_{1}, l_{2}$ and $x_{l}, y_{l}$ so that

$$
\left|a_{1}(0-)-D u^{l_{1}}\left(x_{l}, s_{l}^{1}\right)\right|,\left|b_{1}(0-)-D u^{l_{2}}\left(y_{l}, s_{l}^{2}\right)\right|<\delta / 4
$$

with $x_{l}, y_{l} \in B_{1}(0)$ and $s_{l}^{i} \rightarrow 0^{-}$as $l \rightarrow \infty$. On the other hand, due to (1.1), $u\left(-2^{-m} e_{n}, 0^{-}\right)$exists for any $m$, and thus if $\left|s_{l}^{1}-s_{l}^{2}\right|$ and $\left|s_{l}^{1}\right|,\left|s_{l}^{2}\right|$ is sufficiently small depending on $m$ with $s_{l}^{1}, s_{l}^{2}<0$, then

$$
\frac{\left|u\left(-2^{-m} e_{n}, s_{l}^{1}\right)-u\left(-2^{-m} e_{n}, s_{l}^{2}\right)\right|}{2^{-m}}<\delta / 4 .
$$

Now due to (8.1) and (8.2), if $l$ and $m$ is chosen sufficiently large,

$$
\left|D u\left(-2^{-m} e_{n}, s_{l}^{1}\right)-D u^{l}\left(x_{l}, s_{l}^{1}\right)\right|<\delta / 4
$$

and

$$
\left|D u\left(-2^{-m} e_{n}, s_{l}^{2}\right)-D u^{l}\left(y_{1}, s_{l}^{2}\right)\right|<\delta / 4 .
$$

Putting (8.3)-(8.6) together, one obtains $\left|a_{1}(0-)-b_{1}(0-)\right|<\delta$, a contradiction. 
5. Parallel argument holds for other cases to obtain

$$
a_{1}(0-)=b_{1}(0-)=|D u|\left(x_{0}, t_{0}-\right) \text { and } a_{1}(0+)=b_{1}(0+)=|D u|\left(x_{0}, t_{0}+\right) .
$$

Now it is straightforward from barrier arguments, using the fact that $\left\{u^{l}\right\}$ are viscosity solutions of (HS), to prove that

$$
a_{2}^{\prime}(0-)=b_{2}^{\prime}(0-)=|D u|\left(x_{0}, t_{0}-\right) \text { and } a_{2}^{\prime}(0+)=b_{2}^{\prime}(0+)=|D u|\left(x_{0}, t_{0}+\right),
$$

\section{Global solutions with initially Lipschitz free bound- aries}

As an application of Theorem 1.2, we will show existence, uniqueness and regularity and properties of global solutions of (HS). Let

$$
\Omega_{0}:=\left\{x_{n} \leq g\left(x^{\prime}\right), x=\left(x^{\prime}, x_{n}\right)\right\}
$$

where $g$ is Lipschitz continuous with Lipschitz constant $L<L_{n}$ and $g(0)=0$. Then there is a unique harmonic function $u_{0}$ in $\Omega_{0}$ such that $u_{0}\left(-e_{n}\right)=1$ and $u_{0}=0$ on $\Gamma_{0}$.

Definition 9.1. A lower semi-continuous function $u(x, t)$ in $\mathbb{R}^{n} \times[0, \infty)$ is a global viscosity solution of $(H S)$ with initial data $u_{0}$ if

(a) $u(x, t)$ is a viscosity solution of $(H S)$ in $B_{R}(0) \times(0, \infty)$ for any $R>0$,

(b) $u$ increases in time, and

(c) $u$ locally uniformly converges to $u_{0}$ as $t \rightarrow 0$.

Let $u$ be a global viscosity solution with initial data $u_{0}$. To address uniqueness and regularity properties of $u$, we normalize the solution by assuming that

$$
\lim _{R \rightarrow \infty} \frac{u\left(-R e_{n}, t\right)}{u_{0}\left(-R e_{n}\right)}=1 \text { for each } t \geq 0 .
$$

Lemma 9.2. Suppose $u$ is a global solution of (HS) with (9.1). Then for any $\epsilon>0$ and $T>0$ there exists $R_{0}>0$ such that if $R>R_{0}$

$$
d\left(x, \Gamma_{0}(u) \cap B_{R}(0)\right) \leq \epsilon R \text { for any } x \in \Gamma_{t}(u), 0 \leq t \leq T .
$$


Proof. Denote the following property by $(P)_{t, R}$ :

$$
(P)_{t, R} \quad u \leq \epsilon R^{2} \text { on } \partial B_{R}(0) \times[0, t]
$$

where $\epsilon>0$ is a sufficiently small dimensional constant. Note that by (9.2) and the Harnack inequality, there exists $R_{0}=R_{0}(t)>0$ such that

$$
u\left(x-R e_{n}, t\right) \leq 2 u\left(x-R e_{n}, 0\right) \leq C \epsilon R^{2-\alpha}
$$

for $x \in \Gamma_{0} \cap B_{R}(0)$ and $R \geq R_{0}$.

Now suppose that (9.3) is violated at $t=t_{0}$. This means that for any $\epsilon>0$ there are sequences $R_{k} \rightarrow \infty$ with which $(P)_{t_{0}, R_{k}}$ fails. (Otherwise a barrier argument using radially symmetric barriers will yield (9.3).) In other words, at every $R_{k}=2^{k} R_{0}\left(t_{0}\right)$ there exists $x_{k} \in B_{R_{k}}(0)$ such that $u\left(x_{k}, t_{0}\right) \geq \epsilon R_{k}^{2}$. Since $u$ increases in time, we have

$$
u\left(x_{k}, t\right) \geq \epsilon R_{k}^{2} \text { for } t \geq t_{0} .
$$

Again from a barrier argument it follows that at $t_{1}=t_{0}+\frac{3}{\epsilon}$, the positive phase $\Omega_{t_{1}}(u)$ contains $B_{3 R_{k}}\left(x_{k}\right)$ for any $k$. But then $B_{3 R_{k}}\left(x_{k}\right)$ contains $x=-R_{k} e_{n}$. This and the Harnack inequality contradicts (9.4) if $R_{k}$ is chosen sufficiently large so that $R_{k}>R_{0}\left(t_{1}\right)$ and $\epsilon>>R_{k}^{-\alpha}$.

We now state our main theorem:

Theorem 9.3. There exists a unique global solution $u$ of (HS) with initial data $u_{0}$ which satisfies (9.1). Moreover the following properties hold for $u$ :

(a) For any $t>0, \Gamma_{t}(u)$ is a Lipschitz graph with respect to the direction $e_{n}$ with Lipschitz constant $L$ in $\mathbb{R}^{n}$.

(b) $\Gamma(u) \cap\{t>0\}$ is smooth in space and time.

(c) For any $x \in \Gamma_{0}(u)$ such that $x+d e_{n} \in \Gamma_{t}(u)$,

$$
C^{-1} \leq \frac{\left|D u\left(x+d e_{n}, t\right)\right|}{\left|D u\left(x-d e_{n}, t\right)\right|} \leq C
$$

for a dimensional constant $C>0$.

In addition, if $g\left(-x^{\prime}\right)=g\left(x^{\prime}\right)$, then $u\left(x^{\prime}, x_{n}, t\right)=u\left(-x^{\prime}, x_{n}, t\right)$ and the Lipschitz constant of the graph $\Gamma_{t}(u)$ in any bounded neighborhood in space decreases in time. In particular the Lipschitz constant of $\Gamma_{t}(u)$ at time $t\left(d e_{n} ; u\right)$ in $\left\{x:\left|x_{n}\right| \leq 2^{-l} d\right\}$ is less than $\min \left[L, \frac{C}{l \gamma}\right]$ with $1<\gamma<2$. 
Before proving Theorem 9.3 we first apply the theorem to show that the local solution $u$ given in Theorem 1.2 has a unique blow-up profile at $t=0$ if the subsequence is chosen such that the blow-up limit of the initial data is locally uniform. Let us define

$$
u_{k}(x, t):=\alpha_{k} u\left(\frac{x}{k}, \beta_{k} t\right), \quad \alpha_{k}=u\left(-\frac{e_{n}}{k}, 0\right)^{-1}, \beta_{k}=\frac{\alpha_{k}}{k^{2}},
$$

where $u$ is as given in Theorem 1.2. Note that by definition of viscosity solutions for any $x \in \mathbb{R}^{n}$

$$
|u(x, t)-u(x, 0)| \rightarrow 0 \text { as } t \rightarrow 0 .
$$

Also observe that, since $L_{n}$ is chosen such that $u$ has less than quadratic decay near the free boundary,

$$
\beta_{k}=\left(k^{2} u\left(-\frac{e_{n}}{k}, 0\right)\right)^{-1} \rightarrow 0 \text { as } k \rightarrow 0 .
$$

Therefore for any $t>0$ and sufficiently small $d$

$$
\left|\frac{u_{k}\left(-d k e_{n}, t\right)}{u_{k}\left(-d k e_{n}, 0\right)}-1\right|=\frac{\left|u\left(-d e_{n}, \beta_{k} t\right)-u\left(-e_{n}, 0\right)\right|}{u\left(-d e_{n}, 0\right)} \rightarrow 0 \text { as } k \rightarrow \infty .
$$

Now the second equation Corollary 5.7 applied to $u$ yields that $\Gamma_{t}\left(u_{k}\right)$ is at most $t^{\gamma}$ away from $\Gamma_{0}\left(u_{k}\right)$ in $B_{k}(0)$, where $0<\gamma<1$. Since $\Gamma_{t}(u)$ is Lipschitz continuous in space, it follows from (9.7) with Lemma 2.13 that

$$
\left|\frac{u_{k}\left(-R e_{n}, t\right)}{u_{k}\left(-R e_{n}, 0\right)}-\frac{u_{k}\left(-d k e_{n}, t\right)}{u_{k}\left(-d k e_{n}, 0\right)}\right| \leq \frac{t^{\gamma}}{R}+d^{\gamma} .
$$

if $R<d k$.

Hence for any $\epsilon>0$ there exists $R>0$ such that

$$
\limsup _{k \rightarrow \infty}\left|\frac{u_{k}\left(-R e_{n}, t\right)}{u_{k}\left(-R e_{n}, 0\right)}-1\right| \leq \epsilon
$$

Due to Theorem 1.2 (a), (c) and Corollary 5.7 it follows that $\left\{u_{k}\right\}$ is locally uniformly Hölder continuous in space and $\Gamma\left(u_{k}\right)$ is locally uniformly Lipschitz continuous in space and Hölder continuous in time. Hence by Ascoli-Arzela, along a subsequence $u_{k}(\cdot, t)$ and $d_{\Omega\left(u_{k}\right)}$ locally uniformly converges to $u^{\infty}(\cdot, t)$ and $d_{\Omega^{\infty}}$. It follows that

$$
\left(u^{\infty}\right)^{*}(x, t)=\limsup _{0 \leq s \rightarrow t, y \rightarrow x} u^{\infty}(y, s)
$$


and

$$
\left(u^{\infty}\right)_{*}(x, t)=\liminf _{0 \leq s \rightarrow t, y \rightarrow x} u^{\infty}(y, s) .
$$

It follows from the stability properties of viscosity solutions that $\left(u^{\infty}\right)_{*}$ is a global solution of (HS). (9.1) follows from (9.8) and the local uniforml convergence of $u_{k}$ to $u^{\infty}$. Lastly $u^{\infty}$ increases in time due to the second condition of (1.1). Due to Theorem 9.3 we have the following:

Theorem 9.4. $u^{\infty}$ is the unique global viscosity solution of $(H S)$ with initial positive phase

$$
\Omega_{0}^{\infty}:=\left\{u^{\infty}(\cdot, 0)>0\right\}
$$

satisfying (9.1).

Note that $\Omega_{0}^{\infty}$ is a subsequential limit of $\left\{u_{k}(\cdot, 0)>0\right\}$, i.e., a subsequential limit of the blow-up profile of $\Omega_{0}$ at the origin.

\section{Proof of Theorem 9.3.}

1. Let $\Omega_{0}$ be as given in (9.1). First to show the existence, we consider a sequence $u_{N}$ of star-shaped and Lipschitz initial positive phase $\Omega^{N}$ which coincides with $\Omega_{0}$ in $B_{N^{2}}(0)$, and with fixed boundary data $m_{N}$ on $K_{N}=$ $B_{1}\left(-N e_{n}\right)$ such that $u_{N}\left(-e_{n}, 0\right)=1$. Then due to the main theorem in [CJK], $\left\{u_{N}\right\}_{N}$ is locally uniformly Hölder continuous in space and time, and thus converges locally uniformly to $u$ along a subsequence. It then follows from the construction and the uniform Hölder continuity of $\left\{u_{N}\right\}$ in time that $u$ satisfies (9.1) and (9.2). In addition $u$ is increasing in time and thus a global viscosity solution of (HS).

2. Next we show the regularity of $u$. For given $R>0$, let us define the re-scaled function

$$
u_{R}(x, t):=C(R) R^{-1} u(R x, C(R) t),
$$

where $C(R)=\frac{R}{u\left(-R e_{n}, 0\right)}$. Due to (9.2) it follows that, for any given $T>0$ there exists $R_{0}$ such that

$$
u_{R}\left(-e_{n}, t\right) \in[1,2] \text { for } 0 \leq t \leq \frac{T}{C(R)}, \text { if } R>R_{0} .
$$

Since $u$ is increasing in time, so is $u_{R}$.

Moreover due to (9.3), for given $T>0$ there exists $R_{0}>0$ such that if $R>R_{0}$

$$
\Omega_{T / C(R)}\left(u_{R}\right) \cap B_{1}(0) \subset \Omega_{0}+c_{0} e_{n},
$$

where $c_{0}$ is as given in Theorem 1.2. 
Recall that $\Gamma_{0}\left(u_{R}\right)$ is Lipschitz continuous in $\mathbb{R}^{n}$. with small Lipschitz constant $L<L_{n}$. Due to (9.9) and (9.10), Theorem 1.2 and Corollary 5.6 apply to $u_{R}$ to yield that $\Gamma_{t}\left(u_{R}\right)$ is smooth, (9.5) holds, and is a Lipschitz graph with Lipschitz constant $L+r^{\alpha}$ in $B_{r}(0)$ for

$$
0 \leq t \leq \min \left(\frac{T}{C(R)}, t\left(r e_{n} ; u_{R}\right)\right)
$$

for any $r<r(n)$, where $0<\alpha<1$ depends on $n$.

In terms of $u$, this means that $\Gamma_{t}(u)$ is a Lipschitz graph with Lipschitz constant $L+r^{\alpha}$ in $B_{r R}(0)$ for $0 \leq t \leq \min \left(T, t\left(r R e_{n} ; u\right)\right)$.

Note that, due to Corollary 5.7 and the fact that $L<L_{n}$,

$$
t\left(r R e_{n} ; u\right)>c \frac{(r R)^{2}}{C u\left(-r R e_{n}, 0\right)} \geq c(r R)^{2}
$$

where $c$ is a dimensional constant. Hence if we choose

$$
r=R^{-1 /(1+\alpha / 2)} \text { and } R=T,
$$

then it follows that $\Gamma_{t}(u)$ is a Lipschitz graph with Lipschitz constant $L+$ $R^{-\alpha / 2}$ in $B_{R^{2 \alpha}}(0)$. Since $R$ can be chosen arbitrarily large, we conclude.

3. Now we show the uniqueness of $u$. Suppose $u$ and $v$ are two global solutions satisfying (9.2)-(9.3). For given $R$ and $x \in \mathbb{R}^{n}$, let $\Psi_{R}(x):=$ $\varphi^{-1} \circ \phi \circ \varphi(x)$, where $\varphi(x)=\frac{\epsilon}{R} x$ and $\phi=\psi\left(x+e_{n}\right)-e_{n}$, where

$$
\psi(x)=\frac{1}{|x|^{2}} \cdot\left(x_{1}, \ldots, x_{n-1},-x_{n}+2|x|^{2}\right),
$$

where $x=\left(x_{1}, \ldots, x_{n}\right) \in \mathbb{R}^{n}$. Since $\psi$ is the composition of reflection about $x_{n}=1$ and the conformal mapping sending $x$ to $\frac{x}{|x|^{2}}, \Psi_{R}$ is conformal. $\left(\Psi_{R}\right.$ fixes 0 and will bend $\Gamma(u)$ in the direction of $e_{n}$ by $\epsilon R$ on $\partial B_{R}(0)$ ). We compare $\tilde{u}$ and $v$ in $B_{R}(0) \times[0, T]$, where

$$
\tilde{u}(x, t)=\left(1+2 \epsilon^{1 / 2}\right) u\left(\left(\Psi_{R}\right)^{-1}(x),\left(1+3 \epsilon^{1 / 2}\right) t\right)
$$

Since $1-\epsilon \leq\left|D \Psi_{R}\right| \leq 1+\epsilon$ in $B_{R}(0), \tilde{u}$ is a supersolution of (HS) in $B_{R}(0)$.

Due to (9.3),

$$
\Omega(v) \subset \Omega(u) \text { in }\left(B_{\epsilon^{-1 / 2} R}(0)-B_{R}(0)\right) \times[0, T]
$$

if $R$ is chosen sufficiently large with respect to $\epsilon$ and $T$. Moreover for sufficiently large $R,(9.2)$ and the construction of $\tilde{u}$ yields that

$$
v\left(-R e_{n}, t\right) \leq\left(1-\epsilon^{1 / 2}\right) \tilde{u}\left(-R e_{n}, t\right) .
$$


Recall that both $\Gamma(u)$ and $\Gamma(u)$ are Lipschitz in space, due to our previous argument. Therefore Lemma 2.10 yields that $v \leq \tilde{u}$ in $\partial B_{R}(0)$ as long as $\Omega(v) \subset \Omega(u)$ in $B_{R}(0)$.

Now Theorem 2.7 yields

$$
v \prec \tilde{u} \text { in } B_{R}(0) \times[0, T] .
$$

Since $\epsilon$ can be chosen arbitrarily small and $R \rightarrow \infty$ as $\epsilon \rightarrow 0$, we conclude that $v \leq u$. Similarly $u \leq v$.

4. Finally the last statement of our theorem follows from the uniqueness of global solutions, Theorem 7.2 and the fact that $D u$ is always parallel to $e_{n}$ on the line $\left\{\left|x^{\prime}\right|=0\right\}$.

\section{References}

[C1] L. Caffarelli, A Harnack inequality approach to the regularity of free boundaries, Part I: Lipschitz free boundaries are $C^{1, \alpha}$, Rev.Mat.Iberoamericana, 3 (1987), no. 2, 139-162

[C2] L. Caffarelli, A Harnack inequality approach to the regularity of free boundaries, Part II: Flat free boundaries are Lipschitz,Comm. Pure Appl. Math., 42 (1989), 55-78.

[CJK] S. Choi, D. S. Jerison and I. C. Kim, Regularity for the One-Phase Hele-Shaw problem from a Lipschitz initial surface, Amer. J. Math, 129 (2007), no. 2, 527-582

[D] B. Dahlberg, Harmonic functions in Lipschitz domains, Harmonic analysis in Euclidean spaces, Part 1, pp. 313-322, Proc. Sympos. Pure Math., XXXV, Part, Amer. Math. Soc., Providence, R.I., 1979

[EJ] C. M. Elliot and V. Janovsky, A variational inequality approach to Hele-Shaw flow with a moving boundary, Proc. Roy. Soc. Edinburgh. Sect. A 88 (1981), no.1-2, 93-107.

[ES] J. Escher, G. Simonett, Classical solutions of multidimensional HeleShaw models, SIAM J. Math. Anal 28 (1997), no.5, pp. 1028-1047.

[G] B. Gustaffson, Applications of variational inequalities to a moving boundary problem for Hele Shaw flows, SIAM. J. Math. Anal., 16 (1985), no. 2, 279-300. 
[JK] D. S. Jerison and C. E. Kenig, Boundary behavior of harmonic functions in Non-Tangentially Accessible Domains, Advan. in Math. 46 (1982), 80-147.

[JeKi] D. S. Jerison and I. C. Kim, The one-phase Hele-Shaw problem with singularities, J. Geom. Anal., 15 (2005), 641-667.

[K1] I. C. Kim, Uniqueness and Existence result of Hele-Shaw and Stefan problem, Arch. Rat. Mech. Anal., 168 (2003), 299-328.

[K2] I. C. Kim, Regularity of free boundary in one phase Hele-Shaw problem, J. Diff. equations,223 (2006), 161-184.

[KLV] J.R. King, A.A. Lacey and J.L. Vazquez, Persistence of corners in free boundaries in Hele-Shaw flow, Euro. J. Appl. Math., 6(1995), 455-490.

[S] M. Sakai, Regularity of boundaries of quadrature domains in two dimensions, SIAM J. Math. Anal, 24 (1993), no. 2, 341-364.

[W] K. -O. Widman., Inequalities for the Green function and boundary continuity of the gradient of solutions of elliptic differential equations, Math. Scand., 21 (1967), 17-37. 